\title{
Reducing false rejection rate in iris recognition by quality enhancement and information fusion
}

\author{
Mayank Vatsa \\ West Virginia University
}

Follow this and additional works at: https://researchrepository.wvu.edu/etd

\section{Recommended Citation}

Vatsa, Mayank, "Reducing false rejection rate in iris recognition by quality enhancement and information fusion" (2005). Graduate Theses, Dissertations, and Problem Reports. 1869.

https://researchrepository.wvu.edu/etd/1869

This Thesis is protected by copyright and/or related rights. It has been brought to you by the The Research Repository @ WVU with permission from the rights-holder(s). You are free to use this Thesis in any way that is permitted by the copyright and related rights legislation that applies to your use. For other uses you must obtain permission from the rights-holder(s) directly, unless additional rights are indicated by a Creative Commons license in the record and/ or on the work itself. This Thesis has been accepted for inclusion in WVU Graduate Theses, Dissertations, and Problem Reports collection by an authorized administrator of The Research Repository @ WVU. For more information, please contact researchrepository@mail.wvu.edu. 


\title{
Reducing False Rejection Rate in Iris Recognition by Quality Enhancement and Information Fusion
}

\author{
by \\ Mayank Vatsa \\ Thesis submitted to the \\ College of Engineering and Mineral Resources \\ at West Virginia University \\ in partial fulfillment of the requirements \\ for the degree of \\ Master of Science \\ in \\ Computer Science \\ Afzel Noore, PhD, Chair \\ George Trapp, PhD \\ Raymond Morehead, MD \\ Lane Department of Computer Science and Electrical Engineering \\ Morgantown, West Virginia \\ 2005
}

Keywords: Iris recognition, 1D Log polar Gabor Wavelet, Textural features, Topological features, Quality enhancement, Support Vector Machine, Information fusion 


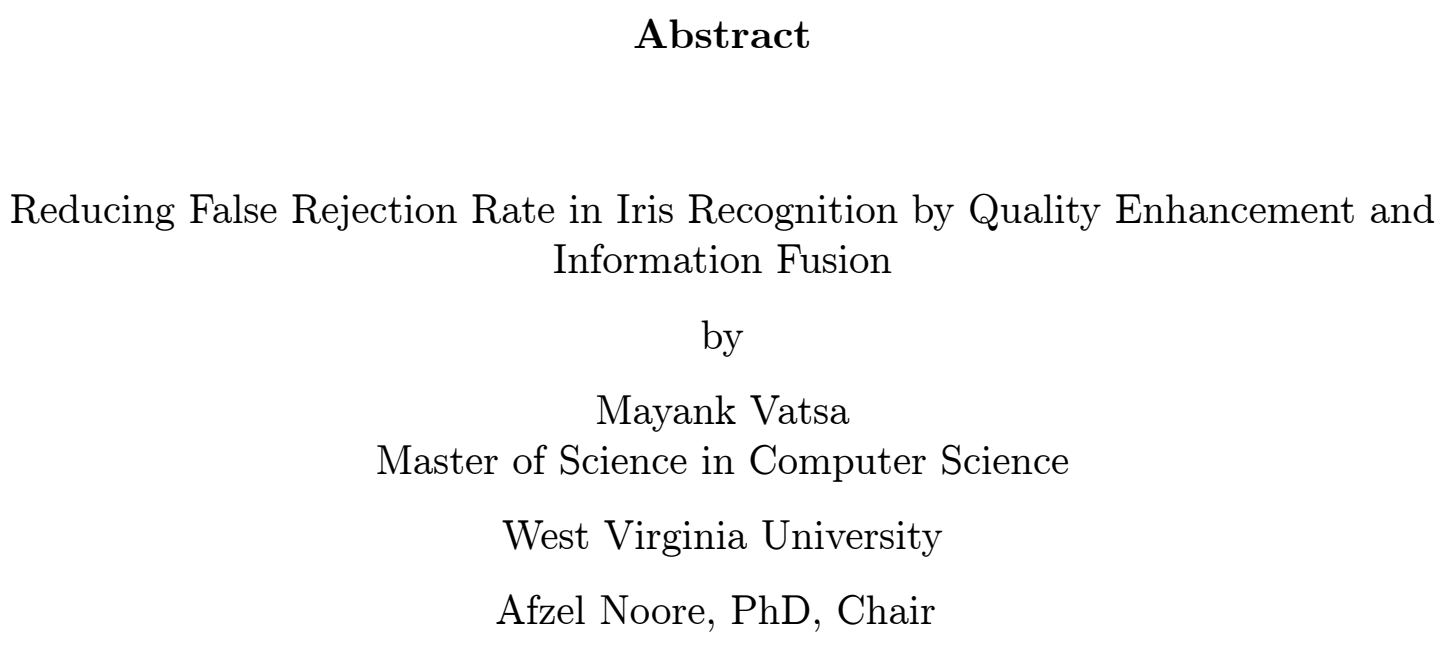

In this thesis we propose a set of algorithms to reduce the false rejection rate of iris recognition. Even though high recognition accuracy is claimed for iris recognition algorithms, high false rejection rates cause the impediment in worldwide use of iris biometrics.

A novel iris segmentation algorithm for non-ideal iris images treating iris as an elliptical object is proposed. Further, quality of the extracted iris image is improved using SVM based enhancement algorithm. In this algorithm, selected enhancement algorithms globally enhance the iris image and the learning algorithm synergistically fuses local information from these intermediate enhanced images. 1D log polar Gabor wavelet is then used to extract the textural features from the enhanced iris image and Euler numbers are used to extract the topological features. The extracted textural features give a global description of the iris image whereas the topological features are rotation, translation and scaling invariant. These two features are fused using the proposed match score and decision fusion algorithms. Among the three proposed fusion algorithm, SVM learning based match score fusion algorithm outperforms other fusion algorithms. Using CASIA, Miles, UBIRIS and UPOL iris databases, experimental results show that the proposed algorithm gives reduced failure to enroll rate with comparable accuracy. 


\section{Acknowledgments}

As I stand at the threshold of earning my master's, I am overwhelmed when I recall all the people who have helped me get this far. First and foremost, I am grateful for the advice and support of my advisor, Dr. Afzel Noore. I have been fortunate in finding an advisor who always had an ear open for the little problems and roadblocks that unavoidably crop up in the course of performing research. His technical and editorial guidance was indispensable to the completion of this thesis and he has taught me immeasurable lessons and insights on the workings of academic research.

I am thankful to my committee members Dr. G. Trapp and Dr. R. Morehead for their supportive approach and helpful suggestions. I also thank Dr. S.K. Singh, Dr. K.K. Shukla, Dr. R.B. Lokesh, Dr. P. Gupta and Dr. P. Mitra for guiding me throughout my bachelor's degree and my stay at the I.I.T. Kanpur. I would also like to thank Dr. A. Ross, Dr. X. $\mathrm{Li}$ and Dr. E. Eschen for making my fundamentals stronger which I used in my research. Acknowledgement is also due to CASIA (China), Miles Research (USA), U.B.I. (Portugal) and Palacky University (Czech Republic) for providing iris databases used in this research. National Institute of Justice is acknowledged for providing funds to carryout my research.

I would like to thank Sushil, Samir, Jidnya, Ashish, Swetha, and Prasad for their help and support. I would also like to thank Lalit, Vineeta, Ravi, and Ratnesh for all what they have given me. It is a great luxury for me to have a friend, Richa, who participated with me in my every success from the first paper to the completion of this thesis. I thank her for all the innovative ideas, long discussions, and night outs.

Finally, from the bottom of my heart, I thank my parent for their sacrifices and all they have done for me. I have been blessed with their love and unwavering support throughout my life. I feel blessed for having them as my parent. As a small token of my appreciation, I am dedicating this thesis to my mother and father. 


\section{Contents}

$\begin{array}{ll}\text { Acknowledgments } & \text { iii }\end{array}$

List of Figures vi vi v v v v v

List of Tables $\quad$ viii

1 Introduction $\quad 1$

1.1 Challenges of Iris Recognition . . . . . . . . . . . . . . . . . 6

1.2 Research Objective .................... . . 8

1.3 Contribution of the Thesis . . . . . . . . . . . . . . . . . . 9

1.4 Organization of the Thesis . . . . . . . . . . . . . . . 10

2 Literature Review of Iris Recognition $\quad 11$

2.1 Literature Review of Iris Recognition . . . . . . . . . . . . . . . . . 11

3 Non-Ideal Iris Image Segmentation and Preprocessing 19

3.1 Proposed Iris Segmentation Algorithm . . . . . . . . . . . . . . . . 20

3.2 Iris Image Quality Enhancement . . . . . . . . . . . . . . . . . . . . . 21

3.2.1 Overview of Support Vector Machine . . . . . . . . . . . . . . . 22

3.2 .2 Generation of Enhanced Quality Iris Image . . . . . . . . . . . . . . . 27

3.2 .3 Image Fusion using $2 \nu$-Support Vector Machine . . . . . . . . . . . . 30

4 Iris Feature Extraction and Matching $\quad 33$

4.1 Iris Texture Template Generation . . . . . . . . . . . . . . . . . . . 33

4.2 Euler Code Generation . . . . . . . . . . . . . . . . . . . . 35

4.3 Feature Matching . . . . . . . . . . . . . . . . . . 37

4.3.1 Iris Code Matching . . . . . . . . . . . . . . . . . . . . 38

4.3 .2 Euler Code Matching . . . . . . . . . . . . . . . . . 39

4.4 Information Fusion . . . . . . . . . . . . . . . . . . . . . . . . . . . . 39

4.4.1 Empirical Decision Fusion . . . . . . . . . . . . . . . . 40

4.4 .2 SVM based Decision Fusion . . . . . . . . . . . . . . . . . . . 43

4.4.3 SVM based Match Score Fusion . . . . . . . . . . . . . . 45 
5 Experimental Results $\quad 47$

5.1 Experimental Results of Proposed Algorithms . . . . . . . . . . . . . . 47

5.2 Performance of Iris Quality Enhancement Algorithm . . . . . . . . . . . . . 52

5.3 Comparison with Existing Algorithms . . . . . . . . . . . . . 56

$\begin{array}{llr}6 & \text { Conclusion } & 58\end{array}$

$\begin{array}{lr}\text { References } & 60\end{array}$ 


\section{List of Figures}

1.1 Different regions of the eye . . . . . . . . . . . . . . . . . 4

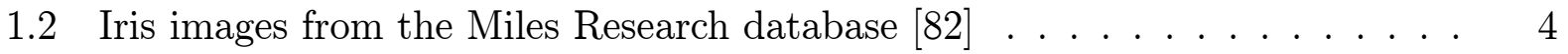

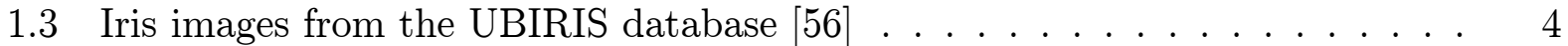

1.4 Iris images from the UPOL database $[22] \ldots \ldots \ldots \ldots$

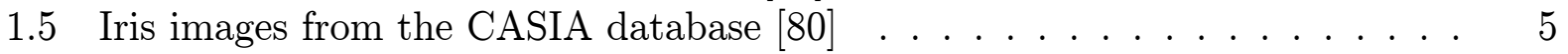

1.6 Iris images from the IITK database [51] . . . . . . . . . . . . . . . . . 5

1.7 Person authentication using iris . . . . . . . . . . . . . . . . . 6

1.8 Example of motion blurness $[56] \ldots \ldots \ldots \ldots$

1.9 Contraction and dilation effect $[56] \ldots \ldots \ldots$. . . . . . . . . . . 7

1.10 Presence of eyelids and eyelashes $[56] \ldots \ldots \ldots \ldots$

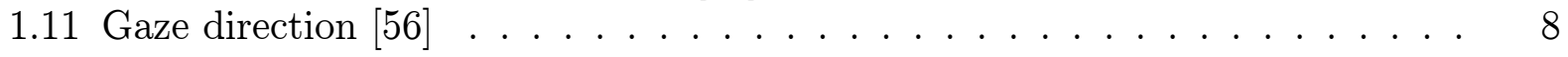

1.12 Images of an individual showing natural luminosity factor $[56] \ldots . . . .8$

2.1 Steps of an iris recognition system . . . . . . . . . . . . . . . 12

3.1 Iris segmentation and unwrapping . . . . . . . . . . . . . . . . . . 21

3.2 A classifier that separates a set of objects into their respective groups with a line . . . . . . . . . . . . . . . . . .

3.3 A classifier that separates a set of objects into their respective groups with a

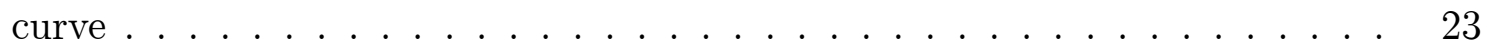

3.4 Mapping non-linearly separable data from the input space to a high dimensional feature space and finding the decision boundary . . . . . . . . . . . 24

3.52 class, 2 feature problem - Non linearly separable data . . . . . . . . . . 28

3.62 class, 2 feature problem - Non linearly separable data becomes linearly

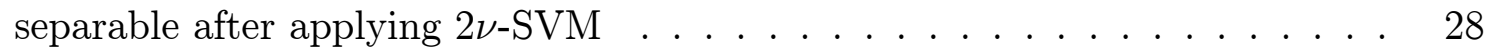

3.7 Iris image enhancement . . . . . . . . . . . . . . . . . . . . . . . . . . 32

4.1 1D log polar Gabor transfer function (a) Linear (b) Logarithmic . . . . . . . 34

4.2 Iris template . . . . . . . . . . . . . . . . 36

4.3 Iris template generation . . . . . . . . . . . . . . . 36

4.4 Generating four binary images from the masked polar image . . . . . . . . 37

4.5 Euler code . . . . . . . . . . . . . . . . . . . . 38

4.6 (a) Database enrollment and (b) Matching strategy . . . . . . . . . . . 42 
4.7 Steps involved in expert fusion . . . . . . . . . . . . . . . . . . . 45

4.8 Steps involved in match score fusion . . . . . . . . . . . . . . . 46

5.1 Iris template generation $\ldots \ldots \ldots \ldots \ldots$

5.2 Iris template generation with eyelids . . . . . . . . . . . . . 48

5.3 Elliptical iris segmentation . . . . . . . . . . . . . . . . . . . . . . 48

5.4 Matching two Euler codes . . . . . . . . . . . . . . . . . . . . 49

5.5 ROC plot showing the performance of proposed algorithms on the CASIA iris

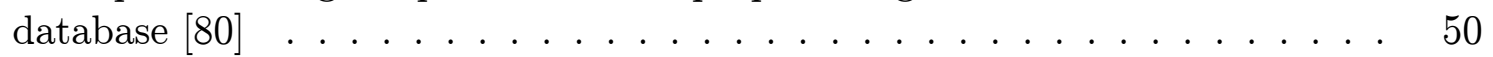

5.6 ROC plot showing the performance of proposed algorithms on Miles iris data-

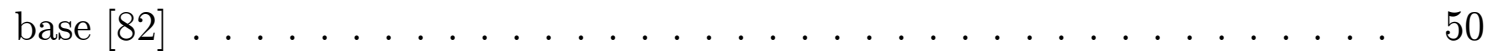

5.7 ROC plot showing the performance of proposed algorithms on UBIRIS iris

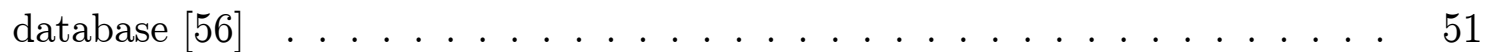

5.8 Sample iris images from UBIRIS database [56] on which the proposed algorithms fail to perform . . . . . . . . . . . . . . . .

5.9 ROC plot showing the performance of proposed algorithms on UPOL iris

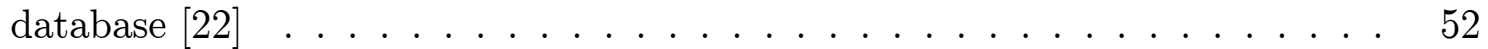

5.10 FRR vs Number of training samples for Daugman's implementation (on CA-

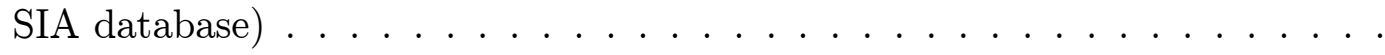

5.11 FRR vs Number of training samples for SVM based match score fusion (on

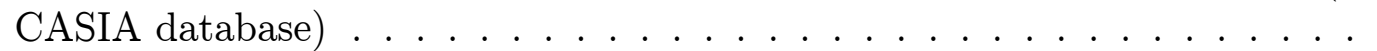




\section{List of Tables}

5.1 Summarizing the performance of proposed algorithm on the four iris databases 53

5.2 Performance of iris recognition algorithms with and without enhancement (Number of training image $=1$ ) . . . . . . . . . . . . 54

5.3 Performance validation of proposed iris enhancement algorithm on different databases (Number of training image $=3$ ) . . . . . . . . . . 56

5.4 Comparison of four iris recognition algorithms on UBIRIS database [56] using three images for training . . . . . . . . . . . . . . . . 57 


\section{Chapter 1}

\section{Introduction}

"Biometrics" means "life measurement", but the term is usually associated with the use of unique physiological characteristics to identify an individual. A biometric system is essentially a multi-disciplinary area of research dealing with fields like pattern recognition, image processing, computer vision, soft computing and artificial intelligence. Biometrics makes personnel identification by determining the authenticity of a specific physiological or behavioral characteristic possessed by the user. Biometrics is thus defined as the "automated method of identifying or authenticating the identity of a living person based on a physiological or behavioral characteristic". By special characteristics we mean the features such as face, iris, fingerprint, and signature. The application which most people associate with biometrics is security and this method of identity verification is preferred over traditional passwords and PIN based methods for various reasons such as:

- The person to be identified is required to be physically present for the identity verification.

- Identification based on biometric techniques obviates the need to remember a password or carry a token.

- It cannot be misplaced or forgotten. 
A biometric system can be either an identification system or a verification (authentication) system depending on the application. Identification and verification are defined below:

Identification - One to Many: Biometrics can be used to determine a person's identity from a database. Identification can be performed without user cooperation in a non-intrusive manner. For example, scanning a crowd with a camera and using face recognition technology, one can determine matches against a known database.

Verification - One to One: Biometrics can be used to verify a person's identity. For example, one can get physical access to a secure area by using finger scans or can get access to a bank account at an ATM by using iris scan. Biometric authentication requires to compare a registered or enrolled biometric sample (biometric template or identifier) against a newly captured biometric sample (for example, the one captured during a login). This is a three-step process. Capture, Process, and Enroll followed by a Verification or Identification process.

During Capture, raw biometric is captured by a sensing device such as a fingerprint scanner or a video camera. The second phase of processing is to extract the distinguishing characteristics from the raw biometric sample and convert them into a processed biometric identifier record (sometimes called biometric sample or biometric template). The next phase is Enrollment, where the processed sample (a mathematical representation of the biometric - not the original biometric sample) is stored/registered in a storage medium for future comparison during authentication. In many commercial applications, we need to store the processed biometric sample only. Original biometric sample may not be reconstructed from this identifier. However, automated capturing and comparison with previously stored data requires the following properties of biometric characteristics:

- Universal: Everyone must have the attribute. The attribute must be one that is universal and seldom lost due to accident or disease.

- Invariance of properties: They should be constant over a long period of time. The attribute should not be subject to significant differences based on age, episodic or chronic disease. 
- Measurability: The properties should be convenient to capture without waiting time and must be easy to gather the attribute data passively.

- Singularity: Each expression of the attribute must be unique to the individual. The characteristics should have sufficient unique properties to distinguish one person from the other. Height, weight, hair, and eye color are all attributes that are unique assuming a particularly precise measure, but do not offer enough points of differentiation to be useful for more than categorizing.

- Acceptance: The capturing should be possible in a way acceptable to a large percentage of the population. Excluded are particularly invasive technologies, i.e. technologies which require a part of the human body to be taken or which (apparently) impair the human body.

- Reducibility: The captured data should be capable of being reduced to a file which is easy to handle.

- Reliability and Tamper-resistant: The attribute should be impractical to masking or manipulation. The process should ensure high reliability and reproducibility.

- Privacy: The process should not violate privacy of the person.

- Comparable: Should be able to reduce the attribute to a state that makes it digitally comparable to others. The less probabilistic the matching involved, the more authoritative the identification.

- Inimitable: The attribute must be irreproducible by other means. The less reproducible the attribute, the more likely it will be authoritative.

Among the various biometric technologies being considered, iris recognition is supposed to be one of the most reliable, universal, measurable, comparable and inimitable. It is a combination of techniques from computer vision, pattern recognition, and the man-machine 


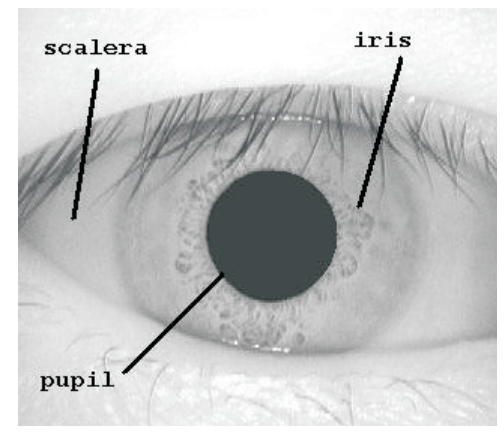

Figure 1.1: Different regions of the eye
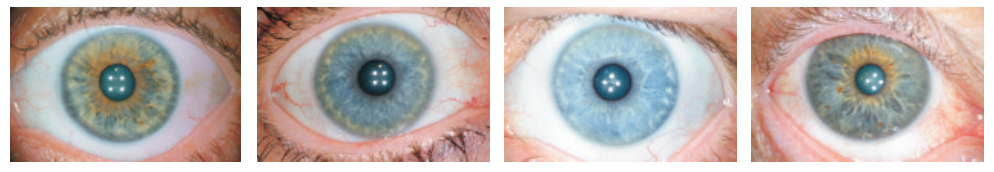

Figure 1.2: Iris images from the Miles Research database [82]

interface. It has been claimed that iris recognition has high confidence for recognizing person's identity by mathematical analysis of random patterns that are visible within the iris of an individual from some distance [19]. Figure 1.1 shows different region of an eye; sclera, iris and pupil. Figure 1.2 to Figure 1.6 shows some examples of iris images. These images are obtained from different databases which are captured using different cameras and lighting conditions. IITK iris database [51], as shown in Figure 1.6, is prepared using visible light and all other databases use the near infra red (IR) light spectrum.
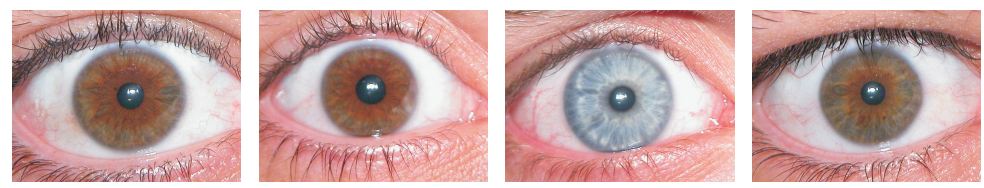

Figure 1.3: Iris images from the UBIRIS database [56]

These images show that the texture of iris changes from person to person. Also, because iris is a protected internal organ whose random texture is stable throughout its life, it serves as a living password that one need not remember but always carries along. Because the randomness of iris patterns have very high dimensionality, recognition decisions are made 


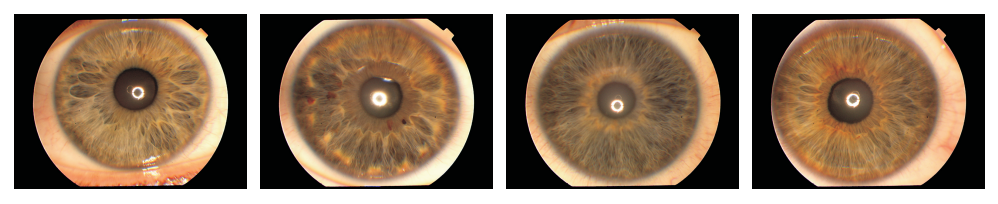

Figure 1.4: Iris images from the UPOL database [22]
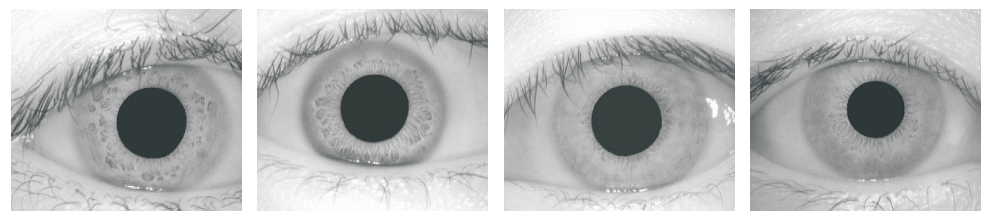

Figure 1.5: Iris images from the CASIA database [80]
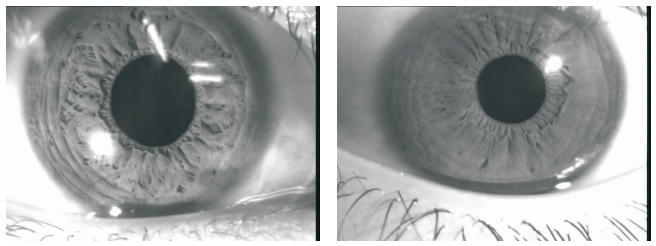

Figure 1.6: Iris images from the IITK database [51]

with confidence levels high enough to support rapid and reliable exhaustive searches through large databases.

The first algorithm of iris recognition was proposed by Flom and Safir [29] in 1985. In 1993, Daugman proposed an iris recognition system representing iris as a mathematical function [17], [19]. After that Wildes [75], Boles [6] and several other researchers proposed different algorithms. A detailed literature survey and analysis of various iris recognition approaches and algorithms is given in Chapter 2 .

For authenticating any person using iris, an input video stream is captured using an IR sensitive CCD camera as shown in Figure 1.7. For capturing the video stream, infrared light is used because blood vessels absorb infra-red light quicker than the surroundings. From this video stream, eye region is captured using an eye localization algorithm. Area of interest (iris) is then detected from eye and the features are extracted. These features are encoded into a pattern using algorithms such as Gabor wavelet based texture encoding. For 


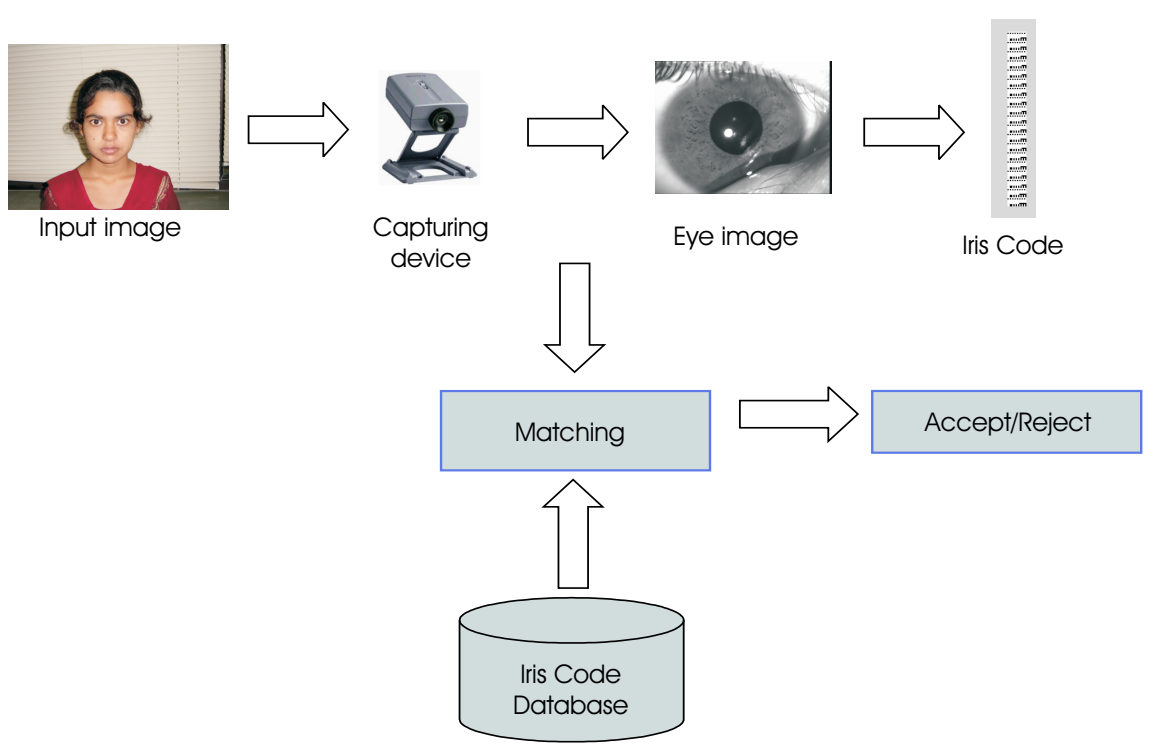

Figure 1.7: Person authentication using iris

enrollment, templates/patterns are stored in the database, and for authentication, encoded patterns from the input iris are matched with those stored in the database using pattern matching algorithm.

\subsection{Challenges of Iris Recognition}

Current iris recognition systems claim to perform with 99.99\% accuracy. Daugman [17] reports the results on a database containing millions of iris images, captured using a high quality near infra-red camera. With such a setup, iris recognition becomes quite an expensive biometric technology. The setup requires user to look into the camera and the image is captured. Many users are not comfortable with the system. Iris images captured from untrained or uncomfortable users suffer due to the quality of the images. If the eyes are not opened properly, some regions of the iris cannot be captured due to occlusion. This affects the process of iris segmentation and consequently feature extraction also. Images may also suffer from motion blurriness, camera diffusion, noise due to transmission, out of focus, presence of eyelids and eyelashes, head rotation, gaze direction, camera angle, reflections, 
contrast, luminosity and problems due to contraction and dilation effects. Figure 1.8 to Figure 1.12 show images with some of the problems mentioned above. These errors in iris images either lead to increase in failure to enroll rate (FER) or decrease the performance of the system due to increase in false rejection rate (FRR).
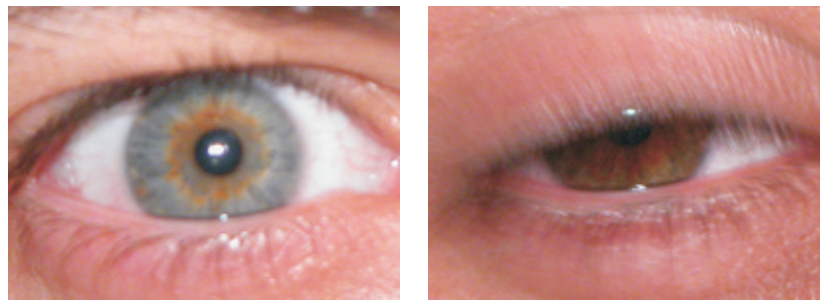

Figure 1.8: Example of motion blurness [56]
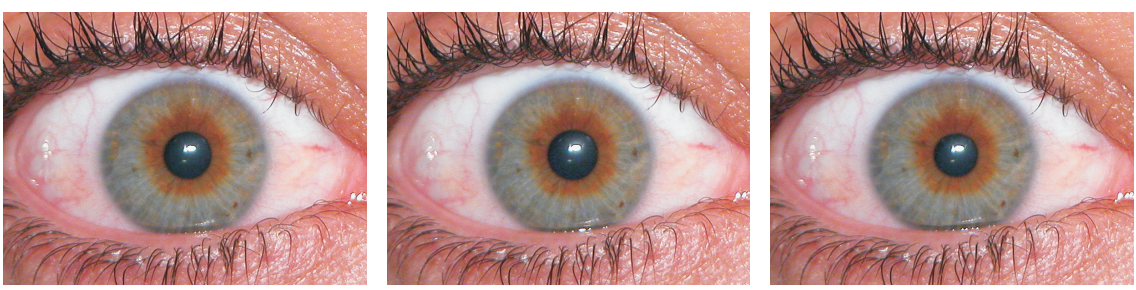

Figure 1.9: Contraction and dilation effect [56]
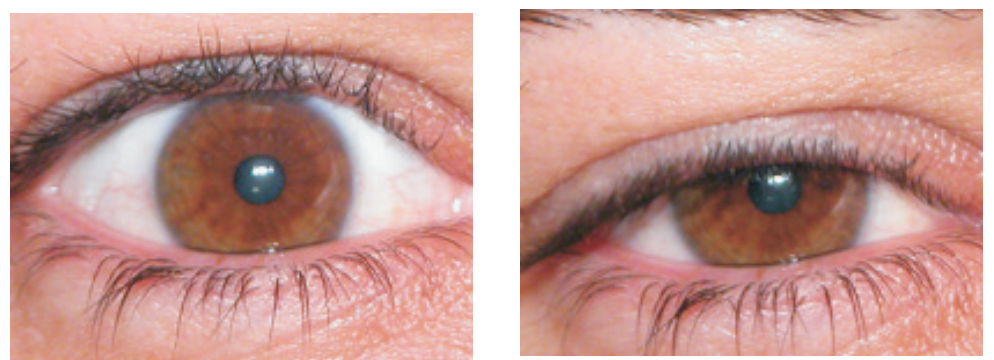

Figure 1.10: Presence of eyelids and eyelashes [56]

Several researchers claim very high performance with a considerable percentage of cases as failure to enroll rate. However, a robust iris recognition algorithm should be able to handle these problems efficiently. Instead of discarding the images as failure to enroll, normalization and preprocessing schemes should be developed to use such images. 

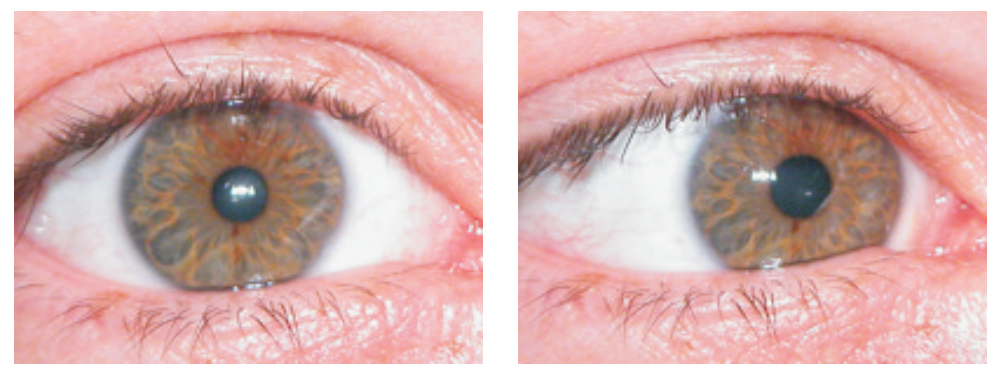

Figure 1.11: Gaze direction [56]
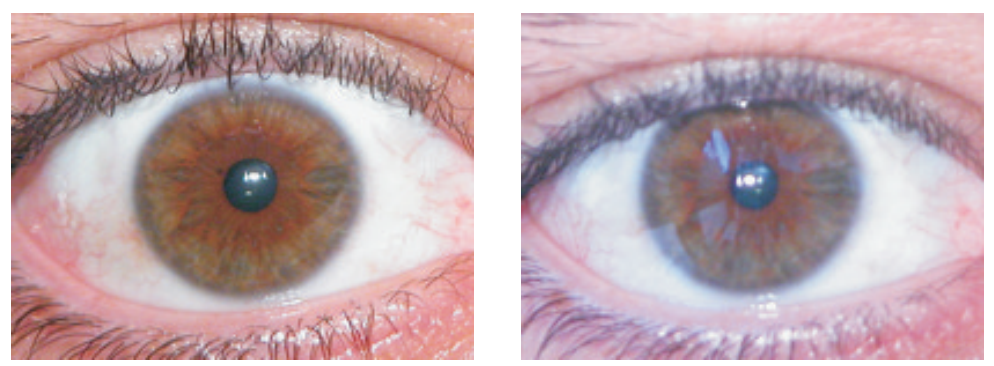

Figure 1.12: Images of an individual showing natural luminosity factor [56]

\section{$1.2 \quad$ Research Objective}

Inspite of the claims for high recognition performance, iris recognition technology still require efforts towards reducing the false rejection and the failure to enroll rates. This research focuses on reducing the high false rejection cases in iris recognition. Thus, our research objectives are:

1. Design an efficient segmentation and quality enhancement algorithm for non-ideal iris images.

2. Design iris recognition algorithm which uses local as well as global features of iris and is invariant to common transformation such as scaling, translation, and rotation.

3. Design fusion algorithm to fuse the information from both local and global features efficiently. 


\subsection{Contribution of the Thesis}

Researchers have presented novel algorithms for detection and preprocessing of iris in presence of eyelids and eyelashes. Considerable work has also been undertaken for feature extraction and matching. Most of the algorithms consider the rich texture of iris and use wavelets to extract and encode the textural features. To analyze the performance of various iris recognition algorithms, we have performed a comprehensive survey of the iris recognition literature.

Based on the literature survey, we found that the quality of iris image plays a major role in the performance of feature extraction and matching. In this research work, we propose an algorithm to enhance the quality of iris images using Support Vector Machine (SVM). SVM learning algorithm learns the 'good' and the 'bad' features of iris image and provides quality enhanced image as the output. This enhancement technique is able to handle problems due to motion blur, camera defocus, natural luminosity factor, brightness, contrast, and background effects.

From the literature survey, we found that the texture based feature extraction algorithms are able to effectively differentiate between iris features from two different classes. The problem arises when features of the same class have variation due to rotation or gaze direction. These variations increase false reject rate of texture based algorithms. Variation in head rotation, contraction, dilation, defocus, and all such challenges do not affect the topology of iris. Thus, for the same person, topological features remain the same in spite of the variations. Considering this observation, we combined the textural and the topological features of iris. Log polar Gabor represents the properties similar to what the visual cortex of human mind represents. So, we have used 1D log polar Gabor for encoding the textural features and Euler number to extract the topological features from the iris.

Further, we proposed decision based fusion and match score based fusion algorithms to combine the textural and the topological features extracted from an iris image. Fusion algorithms learn the performance at different values of matching score and the decisions of Log polar Gabor and Euler number. The performance of quality enhancement, feature 
extraction and fusion algorithms is validated using different databases such as CASIA [80], Miles Research Database [82], UBIRIS [56], and UPOL Iris Database [22]. Using these images, we have established that the proposed algorithms are able to significantly reduce the false rejection rate and the failure to enroll rate in iris biometrics.

\subsection{Organization of the Thesis}

All contributions mentioned above are explained in subsequent chapters. Chapter 2 presents the literature survey of iris recognition. Chapter 3 describes the iris segmentation and quality enhancement algorithm with the overview of Support Vector Machines. Chapter 4 presents the 1D log polar Gabor wavelet based texture feature extraction algorithm and the Euler number based topological feature extraction algorithm. This chapter also describes the three information fusion algorithms to fuse the textural and topological features. Chapter

5 shows the results of quality enhancement, feature extraction and fusion algorithms and Chapter 6 presents the conclusion. 


\section{Chapter 2}

\section{Literature Review of Iris Recognition}

The concept behind iris recognition is similar to fingerprint i.e., every iris has a detailed and unique texture which remain unchanged throughout a person's life and can be used for personal authentication [17]. There are several algorithms proposed by researchers to perform authentication using iris images. This chapter presents the literature review of existing iris recognition algorithms.

\subsection{Literature Review of Iris Recognition}

Since 1985, many researchers have worked on the problem of identifying an individual from iris patterns. It has been discovered that every iris is unique, particularly in the detailed structure of front or anterior layer. Not only are the iris of identical twins different, but the iris of two eyes of the same person are also different. Although specific details of appearance of an iris vary dramatically depending on the level and direction of illumination, the basic, significant features of iris remain extremely stable and do not change over a long time. Even the features which develop over time, such as the atrophic areas, usually develop rather slowly, so that an updated iris image will permit identification for a substantial length of time.

In this section various existing iris recognition algorithms are discussed. As shown in Figure 2.1, the process of iris recognition is divided into four steps. 


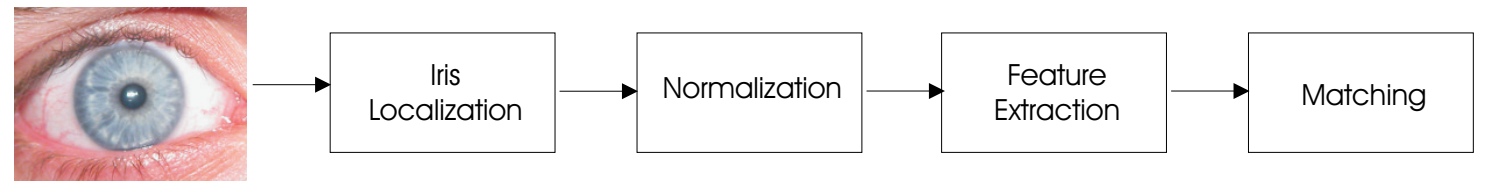

Figure 2.1: Steps of an iris recognition system

- Localization - Inner and outer boundaries of iris are localized.

- Normalization - Iris of different people may vary in size. Even for the same person, size of iris may vary due to contraction and dilation of pupil caused by variation in illumination and other factors. Normalization process ensures that iris patterns of the input image are transformed to facilitate feature extraction and matching.

- Feature extraction - Iris provides abundant texture information. Feature extraction algorithm encodes this textural pattern to form a feature vector.

- Matching - Feature vectors are classified through different matching algorithms such as Hamming distance, weight vector with winner selection, and dissimilarity function.

Most of the researchers have used similar techniques for iris localization and matching. For localization, techniques such as integro-differential operators, hough transform and edge detection are used. Researchers have used different algorithms for extracting features from unwrapped iris images. Depending on the extracted feature, distance measure based matching algorithm are used for matching. Thus in this literature survey, we have focused mainly on the algorithms used for feature extraction.

In 1985, Flom and Safir [29] first proposed an algorithm with imaging hardware for iris recognition. In this algorithm, the eye is first illuminated until the pupil reaches a predetermined size, and an image of the iris and the pupil are captured. This image is then compared with the stored image information for identification. The stored image information is previously obtained from an eye, the pupil of which was similarly captured to the same predetermined size. The illumination of the iris may include oblique illumination from several positions around the circumference of the iris. The illumination from each position may be 
relatively monochromatic, so that the resulting shadow will lack the color of the light source at that position, and provide better contrast for elevation-dependent features. An optical system for performing iris recognition includes a processor which controls an illumination control circuit and a camera to obtain images at several predetermined sizes of the pupil. For matching two preprocessed iris images, a method based on registration and correlation is used.

Daugman [17], [18] proposed the iris recognition algorithm which became the first commercial product [81]. This algorithm is based on the Iris Codes generated using 2D Gabor wavelet. In the preprocessing step, inner and outer boundaries of iris are located using Integro-differential operators. It detects the center and diameter of iris and pupil. Iris region is unwrapped to a rectangular representation to convert it from cartesian to polar coordinates. Feature extraction algorithm uses modified complex valued 2D Gabor wavelets [17], [18]. For matching, Hamming distance (HD) is calculated by using Boolean Exclusive OR operator and perfect match is obtained at hamming distance equal to zero. The algorithm gives an accuracy of more than $99.9 \%$. It is found that the time required for iris identification is less than 1 second.

Wildes made use of an isotropic band-pass decomposition derived by applying Laplacian of Gaussian filters to the image data [75]. Like Daugman, Wilde's also used the first derivative of image intensity to find the location of edges corresponding to the boundaries of iris. Wildes algorithm explicitly models the upper and lower eyelids with parabolic arcs whereas Daugman excludes upper and lower portions of iris. The recognition time taken by the algorithm is also very less.

Boashash and Boles [6] proposed an algorithm based on the zero-crossing of the wavelet transform [50]. They first localized and normalized the iris by using edge detection and other well known computer vision algorithms. Zero-crossings of the wavelet transform are then calculated at various resolutions over concentric circles on the iris. Resulting one dimensional (1D) signals are then compared with the model features using different dissimilarity function. The algorithm can handle noisy conditions as well as variations in illumination. It is also 
invariant to translation, rotation and scale. A similar type of system has been presented in [3] which is based on the zero-crossing discrete dyadic wavelet transform representation and shows a high level of accuracy.

In [35], another algorithm was proposed to extract the features of iris by Multi-resolution Independent Component Identification (M-ICA). M-ICA provides good representation of signals with time and frequency. It is used to extract features of iris for matching using conventional algorithms. Accuracy obtained is low because the M-ICA does not give good performance on class-separability.

Dobes et al. [21], used the mutual information between two iris images to determine if they are from the same eye. Iris boundary is searched by reducing the image resolution to $1 / 5$. Gaussian convolution mask is applied to emphasize the boundary edges and suppress unwanted details. Outer boundary edge gives the parameters of the outer circle of iris. Several horizontal and vertical lines are drawn and every crossing with the outer boundary gives two crossing points for the next computation of iris radius. Inner radius is calculated as mean of the distances between the crossing points and the center. For iris recognition, mutual information based on intensity values and their positions is used for alignment and verification. Authors report the best accuracy of $99.05 \%$ at a resolution scale of 0.2 .

Iris recognition algorithm developed by Ganeshan [31] et al. localizes iris by first forming a circular contour around the eye to eliminate redundant parts. Image processing techniques such as thresholding and gray level slicing are used to detect the pupillary boundary. For recognition, first the limbus diameter and the pupil diameter are determined. If the two ratios match, an isotropic circularly symmetric band pass decomposition is obtained from the application of Laplacian of Gaussian filter to the image. A pyramid of the iris image is formed and matching is performed using normalized correlation technique.

Quality assessment of iris images can reduce the errors caused due to bad samples. Tan et al., [64] use total spectrum power and ratio of middle frequency power to other frequency power of iris image as the two features to assess the quality of the iris image. SVM is trained using these two features to predict the quality of the input iris images. The images which pass 
this quality assessment test are localized using projection and Hough transform. Localized iris is unwrapped to a rectangular block of fixed size and then subjected to lighting correction for enhancement. For feature extraction, a bank of spatial filters is defined whose kernels are suitable for iris recognition. This filter bank captures the local details of iris to generate discriminating textural features. Dimensionality reduction of the features is performed by applying Fisher Linear Discriminant analysis and then the nearest neighbor classification is adopted for classification.

In another research work, Ma et al., [45] used the intuition that for iris images, it is difficult to segment and locate blocks in gray level images due to the irregular structure of iris images. Thus they used the position of local sharp variation points as features. Iris is detected from the background using projection and canny edge detector and is then unwrapped to a rectangular block of fixed size. This unwrapped iris image is subjected to lighting correction and histogram equalization for enhancement. 1D signals generated from the $2 \mathrm{D}$ iris image are then convolved with dyadic wavelet transform for local extreme detection. The occurrence of local minima and maxima are encoded to form a feature vector and matched using exclusive OR operation. Authors report an Equal Error Rate (EER) of 0.09\% on CASIA iris image database. In another paper [46], a 1D intensity signal is constructed containing the majority of local variations of the iris. Gaussian-Hermite moments of such intensity signals are used as distinguishing features. Dimensionality reduction is performed in the same manner as [64] and finally the features are classified using Nearest Neighbor Classifier. Authors report an accuracy of $99.61 \%$ on the CASIA database with reduced time complexity.

Textural representation provides global description of the iris texture which works well for the noisy images, geometric and photometric deformations. Local key intensity variations provide local description of the features with fine spatial changes in iris patterns. Thus an optimal classifier would be the combination of both. Sun et al. in [62] used the concept to develop an elastic blob matching algorithm to overcome the limitations of local classifiers. For moment based blob matching, blocks of interest are segmented from the unwrapped iris 
by applying Dyadic Wavelet Transform. These blocks are represented by their geometric moments and aligned for rotation, translation and scale. Matching is also performed based on these parameters. On CASIA database, authors claim that the performance of their algorithm is better than the Daugman's algorithm [17].

Most of the algorithms extract features from the unwrapped iris to account for the deformation and reduce the complexity. Avila and Reilo [4] used the unwrapped polar iris along with the detected circular iris region for feature extraction and matching. Before, unwrapping, superior and inferior cones are eliminated from the segmented iris. Unwrapped iris is weighted with the imaginary part of Gabor filter in four orientations. For feature extraction from the virtual iris circle, first iris signatures are generated. Iris signature is gray level values on the contour of a virtual circle which is centered at the centroid of pupil, with fixed ratio and certain angular increments. Another signature is generated in terms of gray level values along the contours of virtual circle from inner to outer radius. Unique features are extracted from these signature codes and represented using discrete dyadic wavelet transform - quadratic spline of compact support. For classification and verification, they used three metrics, Euclidean distance, binary hamming distance and $d_{j}$ which is directly related with the zero-crossing representation of a 1D signal. Authors report the best accuracy of $97.6 \%$ for the iris signature from virtual circle and $99.6 \%$ for the signature from annular region. This accuracy is obtained using hamming distance as the classification metric.

Other researchers have used different algorithms for feature extraction. Dargham et al. [14] used thresholding to detect iris from pupil and the surroundings. Detected iris is then transformed into a rectangular format. Self organizing map networks are then used to recognize the iris patterns and an accuracy of around $83 \%$ is achieved. In another algorithm by Ma et al. [44], circular symmetry filters are used to capture local texture information of the iris, which are then used to construct a fixed length feature vector. Nearest feature line algorithm is used for iris image matching. Results obtained were $0.01 \%$ for false match and $2.17 \%$ for false non-match rate. Chen and Yuan [8] developed an algorithm for extracting the iris features based on fractal dimension. Iris zone is partitioned into small blocks in which 
local fractal dimension features are computed as the iris code. Finally the patterns are matched using k-means and neural networks. The results obtained are $91.8 \%$ acceptance for authentic person and $100 \%$ rejection rate for impostors. Wang et al. [76] used Gabor filters and 2D wavelet transforms for feature extraction. For identification, weighted Euclidean distance classification is used. This algorithm is invariant to translation and rotation and tolerant to illumination. The classification rate using Gabor is $98.3 \%$ and the accuracy with wavelets is $82.51 \%$. Tisse et al. [66] proposed an algorithm for localization and extraction of iris. For localization, combination of integro-differential operators with Hough Transform is used and for feature extraction, the concept of instantaneous phase or emergent frequency is used. Iris code is generated by thresholding both the models of the emergent frequency and the real and imaginary parts of the instantaneous phase. Finally, matching is performed using Hamming distance. In this algorithm, false rejection rate of $11 \%$ was achieved. Lim et al. [42] used Haar Wavelet transform to extract features from iris images. By applying the transform four times on an image of size $450 \times 60$ and combining the features, an 87-bit feature vector is obtained. This feature vector is the compact representation of iris image. For classification of feature vectors, weight vector initialization and winner selection strategy are used. The recognition rate obtained is around 98.4\%. Machala and Pospisil proposed two new algorithms for statistical and computer evaluations of the iris structure in [47]. The algorithms are partly based on the correlation analysis and partly on the median binary code of commensurable regions of digitized iris image. Similarly, the algorithm of iris structure characterization using statistical and spectral analysis of color iris images is considered in [33]. They used the Wiener spectra for characterization of iris patterns. Petr et al. explained human iris structure and classified using coherent Fourier spectra of the optical transmission, [20], [55].

An efficient iris recognition algorithm is described in [53]. It is based on an empirical analysis of the iris image. The algorithm is split in several steps: capturing iris patterns; determining the location of iris boundaries, converting the iris boundary to the stretched polar coordinate system; extracting the iris code based on texture analysis using wavelet 
transforms; and classification of the iris code. This algorithm uses wavelet transforms for texture analysis, and relies on knowledge of the general structure of human iris. Algorithm has been implemented and tested using a dataset of 240 samples of iris data with different contrast quality.

There are several research papers that proposed different algorithms for feature extraction such as fractal analysis [8], orthogonal wavelet transformations [60], ICA and PCA [23], correlation filters [73], Zak's transform to Gabor expansion [12], Fourier transform, circular and radial features [51] and many others [78], [79]. There are other research papers that have proposed algorithms for synthetic iris image generation to generate large scale iris databases [11], [41], [48], [77]. In a study [59] it has also been suggested that after eye surgery there is a need to update the iris database as some of the features do change due to surgery. Iris recognition is also used in multimodal biometrics [71], [74], biometric watermarking for template protection [70], [71], and other application areas. 


\section{Chapter 3}

\section{Non-Ideal Iris Image Segmentation and Preprocessing}

First step of iris recognition is to detect the pupil and the iris boundaries from input eye image and unwrap the extracted iris in a rectangular form. It also involves preprocessing of the iris image for normalization and scale invariance. However, when images are captured under uncontrolled environment, non-ideal iris images can result. Non-ideal iris and pupil are elliptical in shape and may vary in quality depending on how the image is captured. Detection of iris and pupil boundaries in such cases is a challenging task.

Researchers have proposed different algorithms to detect the iris boundaries. Daugman [17] has used integro-differential operator to locate inner and outer boundaries of the iris. Wildes [75] also used the first derivative of image intensity to find the location of edges corresponding to the borders of iris. The algorithm explicitly models the upper and the lower eyelids with parabolic arcs. Boashash and Boles [6] used edge detection techniques to detect and localize the iris. Tisse et al. [65] used a combination of integro-differential operators with Hough Transform for localization. Most of the detection algorithms relied on the circular structure of iris. However, Chun and Chung [10] considered iris to be an ellipse and applied integro-differential operator to detect the inner and the outer boundaries. Sung et al. [63] proposed that the area between inner and outer boundary contains some redundant information. To remove this redundant information, collarette boundary is detected along 
with the inner and the outer boundaries. Features are extracted from the region between inner boundary and the collarette boundary by applying SVM in wavelet domain. Authors report an accuracy of $80.49 \%$ with the features extracted from the region in between the inner and the outer boundary and the performance is increased to $100 \%$ when only the region between the inner and the collarette boundary is considered.

\subsection{Proposed Iris Segmentation Algorithm}

In this research, to identify the boundary of pupil in non-ideal conditions, an elliptical region with major axis $a$, minor axis $b$, and center $(x, y)$ is selected in the center of eye and the intensity values for a certain number of points on the circumference are computed. Parameters of the ellipse $(a, b, x, y, \theta)$ are iteratively varied to increase the size of ellipse and every time the same number of points are randomly chosen on the circumference to calculate the total intensity value. This process is repeated to find the boundary with maximum variation in intensity and the center of pupil. Outer boundary of the iris is detected in a similar manner. Parameters for outer boundary $a, b, x, y$ and $\theta$ are varied with the initial parameters equal to the pupil boundary parameters. A certain number of points are chosen on the circumference and sum of the intensity values is computed. Values corresponding to the maximum intensity change gives the outer boundary of iris and the center of this ellipse gives the center of iris. Thus the center of pupil, center of iris, and major and minor axis are determined. An ellipse is drawn using these values to locate the pupil and the iris edges.

Eyelids and eyelashes are isolated from detected iris image because they degrade the performance of the system. Eyelids are isolated by first fitting a line to the upper and the lower eyelid using linear Hough transform. A horizontal line is drawn which intersects with the first line at the iris edge that is closest to the pupil. A second horizontal line allows the maximum isolation of eyelid regions. Canny edge detection is used to create the edge map, and only the horizontal gradient information is used. If the maximum value in Hough space is lower than a set threshold then no line is fitted, since this corresponds to non-occluding eyelids. Also, the lines are restricted to lie exterior to the pupil region, and interior to the iris 
region. A similar process is followed for detecting eyelashes. A mask based on the detected eyelids and eyelashes is then used to extract the iris without noise. Image processing of the iris is computationally intensive as the area of interest is of donut shape and grabbing the pixels in this region requires repeated rectangular to polar conversion. To simplify this, the iris is first unwrapped into a rectangular region and then converted to polar coordinates. An example of iris segmentation algorithm is shown in Figure 3.1.

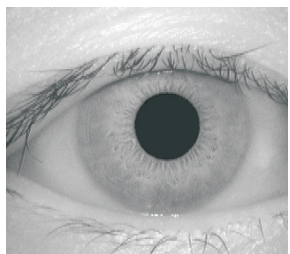

(a) Original image

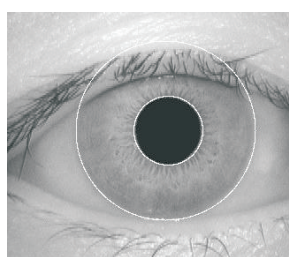

(b) Pupil and iris detected

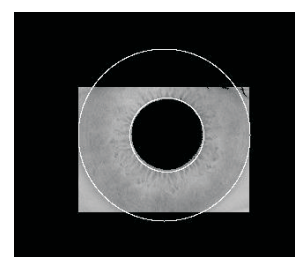

(c) Masked iris image

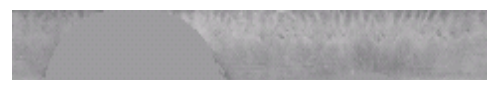

(d) Unwrapped iris

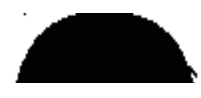

(e) Corresponding mask

Figure 3.1: Iris segmentation and unwrapping

\subsection{Iris Image Quality Enhancement}

Non-ideal images collected in an uncontrolled environment may require the application of specific algorithms to local regions that need enhancement. However, identifying and isolating these local regions in an iris image can be tedious, time consuming, and not pragmatic. There are several research papers which propose algorithms for image quality enhancement. In [34], authors have used Gabor filters to detect the noise present in images. They further proposed the use of inpainting to remove noise influence and estimate occluding pixels. For validation, images are taken from the CASIA database and matched using Daugman's algorithm [17]. Jang et al., [36] proposed an algorithm for iris image quality assessment. Images of left and right eye are captured and detected for counterfeit and noisy data using 2D bisection based Hough Transform, region based template deformation, followed by masking in 
2D Fast Fourier Transform (FFT) of the eye. Features are extracted from the classified good quality data using Daubechies Wavelet and then matched using Support Vector Machines (SVM) and Euclidean distance. Authors report an increase in performance after using the quality assessment algorithm. Kong and Zhang [40] developed a noise detection model for accurate iris segmentation which is divided into two parts, eyelash detection and reflection detection. The value of convolution with 1D Gabor and variance of intensity are used to separate the eyelashes. Similarly, weak and strong reflections are classified using mean and standard deviation. Strong reflection represents the region with higher intensity and weak reflection represents the transition from strong reflection to iris. They show an improvement in the performance of iris recognition using 2D Gabor with the proposed detection model.

For quality enhancement, first we concurrently apply a set of selected enhancement algorithms globally to the whole image. Thus each image contains enhanced local regions. The enhanced pixels of local regions from each of the transformed images are identified using a Support Vector Machine (SVM) based learning algorithm. Fusion of these high quality pixels is synergistically combined to form a single high quality iris image. In the next subsection, an overview of Support Vector Machine is presented and then the proposed iris quality enhancement algorithm is described in subsection 3.2.2.

\subsubsection{Overview of Support Vector Machine}

Support Vector Machine (SVM) proposed by Vapnik [67] is a powerful methodology for solving problems in nonlinear classification, function estimation and density estimation. The concept of decision planes that define decision boundaries is the basis of SVM. A plane that separates between a set of objects belonging to different classes is called the decision plane. Figure 3.2 shows a simple example of linear classifier separating the objects belonging to two different classes, Class 1 and Class $_{2}$ using a decision plane. A separating line defines the boundary that classifies objects on the two sides; all objects on one side of the line belong to Class $_{1}$ and all objects on other side of the line belong to Class. $s_{2}$. Any new object (test sample) falling to the right is classified as $\mathrm{Class}_{2}$, otherwise it is classified as Class 1 . 


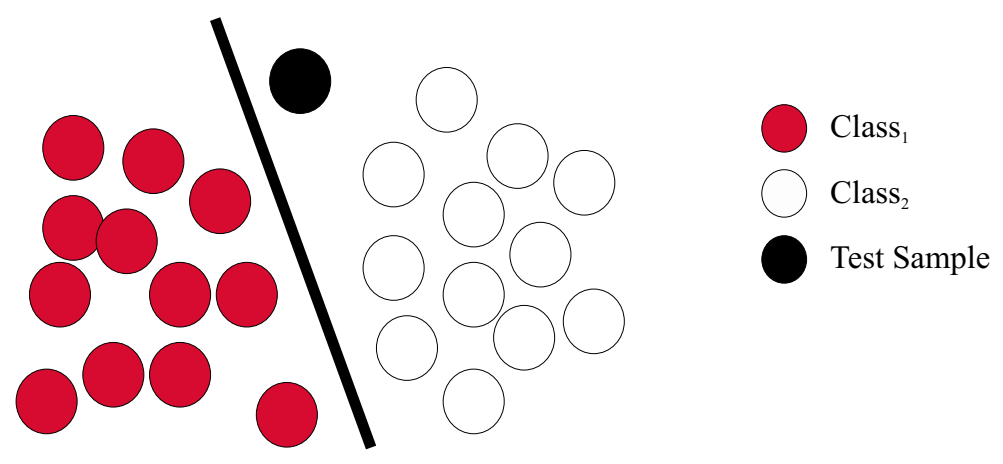

Figure 3.2: A classifier that separates a set of objects into their respective groups with a line

In general, classification tasks are not simple. Often more complex structures are required to make an optimal separation and correctly classify new objects (test samples) on the basis of training samples. Figure 3.3 shows an example of complex classification task in which the decision boundary is a complex curve rather than a straight line. Support Vector Machines are particularly suited to handle such tasks.

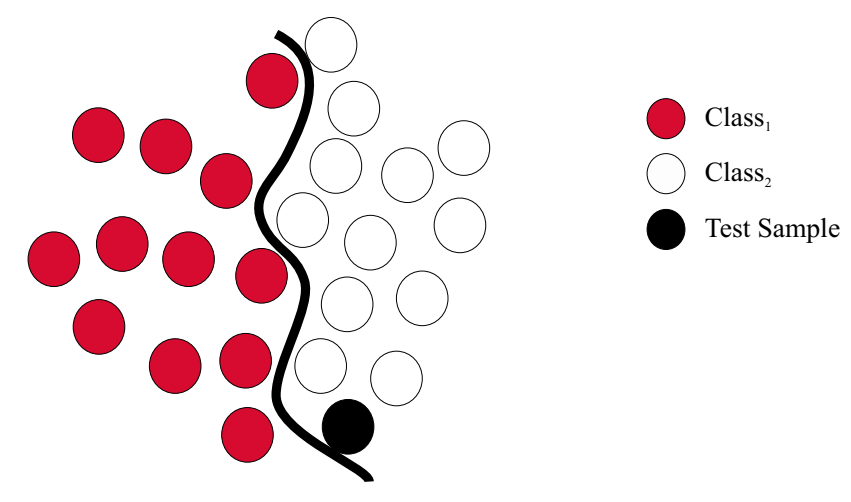

Figure 3.3: A classifier that separates a set of objects into their respective groups with a curve

SVM starts from the goal of separating data with a hyperplane and extending this to nonlinear decision boundaries. Figure 3.4 shows the basic idea behind Support Vector Machines. In this, original objects are mapped (using mathematical functions) into a new feature space such that it is now easy to separate the mapped objects in the new feature space. This process of rearranging the objects is known as mapping or transformation.

SVM is thus a classifier that performs classification tasks by constructing hyperplanes in 


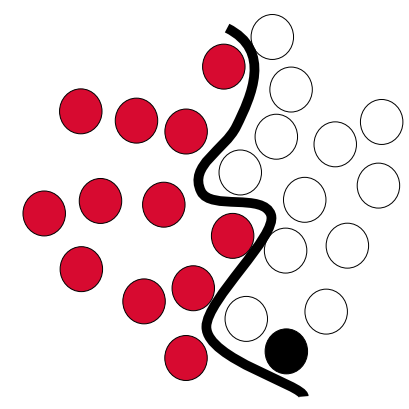

Input Space

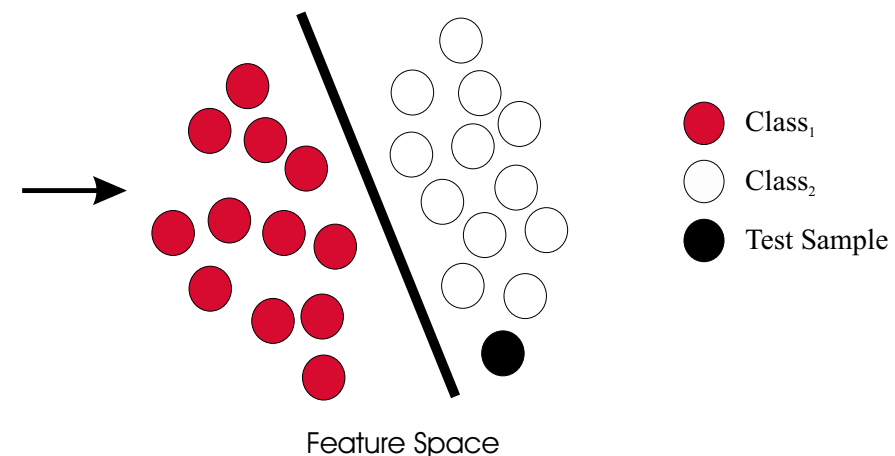

Feature Space

Figure 3.4: Mapping non-linearly separable data from the input space to a high dimensional feature space and finding the decision boundary

a multidimensional space that separates the data points into different classes. To construct the optimal hyperplane, SVM uses an iterative training algorithm to minimize the error function. Rest of the section describes the mathematical formulation of non-linear SVM which is more useful in terms of practical biometrics.

Sometimes the training data points are not clearly separable and are characterized as fuzzy separable data. If we think from the biometrics perspective, fuzzy data are more common and SVM which deals with such data can provide the user with the probabilistic recommendation. A two layer network can provide an adequate flexibility in the classification of fuzzy separable data. This network can be designed by inserting a well designed nonlinear hidden-layer between input and output. The original linearly non-separable data can be mapped into a new feature space, i.e., a new space represented by hidden nodes such that the mapped patterns become linearly separable.

Let $\varphi_{i}(x)$ be a nonlinear function representing hidden nodes, where $i=1, \ldots, N$ is a generalized decision function and can be written as,

$$
f(x)=\sum_{i=1}^{N} w_{i} \varphi_{i}(x)+b=W \bar{\varphi}(x)+b
$$

where $\bar{\varphi}(x)=\left[\varphi_{1}(x), \varphi_{2}(x), \ldots, \varphi_{N}(x)\right]^{T}$. To obtain a nonlinear decision boundary and enhanced discrimination power, we can rewrite the above equation as 


$$
f(x)=\sum_{i=1}^{N} y_{i} \alpha_{i} K\left(x, x_{i}\right)+b
$$

where $K\left(x, x_{i}\right)$ is a nonlinear kernel which enhances the discrimination power and $\alpha_{i}$ is the Lagrange multiplier. Basic idea behind nonlinear SVM is to use a kernel function $K\left(x, x_{i}\right)$ to map the data of input space to feature space such that the mapped data becomes linearly separable in the feature space. One example of such kernel is the $R B F$ kernel

$$
K\left(x, x_{i}\right)=\exp \left[\frac{\left\|x-x_{i}\right\|^{2}}{2 \sigma^{2}}\right]
$$

The Lagrange multipliers $\alpha_{i}$ are determined by maximizing $L(\alpha)$ to $\sum_{i=1}^{N} \alpha_{i} y_{i}=0$ and $0 \leq \alpha \leq C, i=1, \ldots, N$ where

$$
L(\alpha)=\sum_{i=1}^{N} \alpha_{i}-\frac{1}{2} \sum_{i=1}^{N} \sum_{j=1}^{N}\left(\alpha_{i} \alpha_{j} y_{i} y_{j} K\left(x_{i}, x_{j}\right)\right)
$$

and $C$ is a factor used to control the violation of the safety margin rule [68].

In practice, if highly noisy training data is present then finding a separating hyperplane is not a trivial task. Moreover, setting of the error penalty in the objective function is based on trial and error, which requires additional time consuming training. One possible way to solve this problem is the use of $\nu$-SVM of a soft margin variant of the optimal hyperplane which uses the $\nu$-parameterization [7]. Here the parameter $C$ is replaced by another parameter $\nu \epsilon[0,1]$ which is the lower and the upper bound on the number of examples that are support vectors and that lie on the wrong side of the hyperplane, respectively. Further, dual $\nu$-SVM [9] is designed to reduce the complexity of error penalty selection and improve the performance in computation and classification. Dual $\nu$-SVM $(2 \nu$-SVM $)$ is briefly described as follows: 
Let $\left\{x_{i}, y_{i}\right\}$ be a set of $N$ data vectors with $x_{i} \epsilon \Re_{d}, y_{i} \epsilon(+1,-1)$, and $i=1, \ldots, N . x_{i}$ is the $i^{\text {th }}$ data vector that belongs to binary class $y_{i}$. The objective of training $2 \nu$-SVM is to find the hyperplane that separates two classes with widest margins, i.e.,

$$
w(x)+b=0
$$

subject to,

$$
y_{i}(w \varphi(x)+b) \geq\left(\rho-\psi_{i}\right), \psi_{i} \geq 0
$$

to minimize,

$$
\frac{1}{2}\|w\|^{2}-\sum_{i} C_{i}\left(\nu \rho-\psi_{i}\right)
$$

where $\rho$ is the position of margin and $\nu$ is the error parameter. $\varphi(x)$ is the mapping function used to map data space to the feature space, and provide generalization for the decision function that may not be a linear function of the training data. $C_{i}\left(\nu \rho-\psi_{i}\right)$ is the cost of errors, $w$ is the normal vector, $b$ is the bias, and $\psi_{i}$ is the slack variable for classification errors. $\nu$ is the error parameter that can be calculated using $\nu_{+}$and $\nu_{-}$, which are the error parameters for training the positive and negative classes respectively.

$$
\nu=\frac{2 \nu_{+} \nu_{-}}{\nu_{+}+\nu_{-}}, \quad 0<\nu_{+}<1 \text { and } 0<\nu_{-}<1
$$

Error penalty $C_{i}$ is calculated as,

$$
C=\left\{\begin{array}{lll}
C_{+}, & \text {if } & y_{i}=+1 \\
C_{-}, & \text {if } & y_{i}=-1
\end{array}\right.
$$

where,

$$
C_{+}=\left[n_{+}\left(1+\frac{\nu_{+}}{\nu_{-}}\right)\right]^{-1}
$$




$$
C_{-}=\left[n_{-}\left(1+\frac{\nu_{-}}{\nu_{+}}\right)\right]^{-1}
$$

and $n_{+}$and $n_{-}$are the number of training points for the positive and the negative classes. $2 \nu$-SVM training can be formulated as,

$$
\max _{\left(\alpha_{i}\right)}\left\{-\frac{1}{2} \sum_{i, j} \alpha_{i} \alpha_{j} y_{i} y_{j} K\left(x_{i}, x_{j}\right)\right\}
$$

where,

$$
\begin{aligned}
& 0 \leq \alpha_{i} \leq C_{i} \\
& \sum_{i} \alpha_{i} y_{i}=0 \\
& \sum_{i} \alpha_{i} \geq \nu
\end{aligned}
$$

$i, j \in 1, \ldots, N$ and kernel function is

$$
K\left(x_{i}, x_{j}\right)=\varphi\left(x_{i}\right) \varphi\left(x_{j}\right)
$$

Here the kernel function $K\left(x_{i}, x_{j}\right)$ is the RBF kernel as described in Equation 3-12. $2 \nu$-SVM is initialized and optimized using iterative decomposition training [9], which leads to the reduced complexity. If $n$ is the set of data vectors, then the complexity without optimization is $O\left(n^{2}\right)$ and with optimization is $O(n)$ [9]. Now consider a 2 class, 2 feature problem as shown in Figure 3.5. By applying $2 \nu$-SVM the nonlinearly separable data is mapped in a higher dimensional feature space where the data becomes linearly separable as shown in Figure 3.6.

\subsubsection{Generation of Enhanced Quality Iris Image}

For every iris image in the training database, a set of transformed images are generated by applying standard enhancement algorithms for noise removal, defocus and motion blur re- 


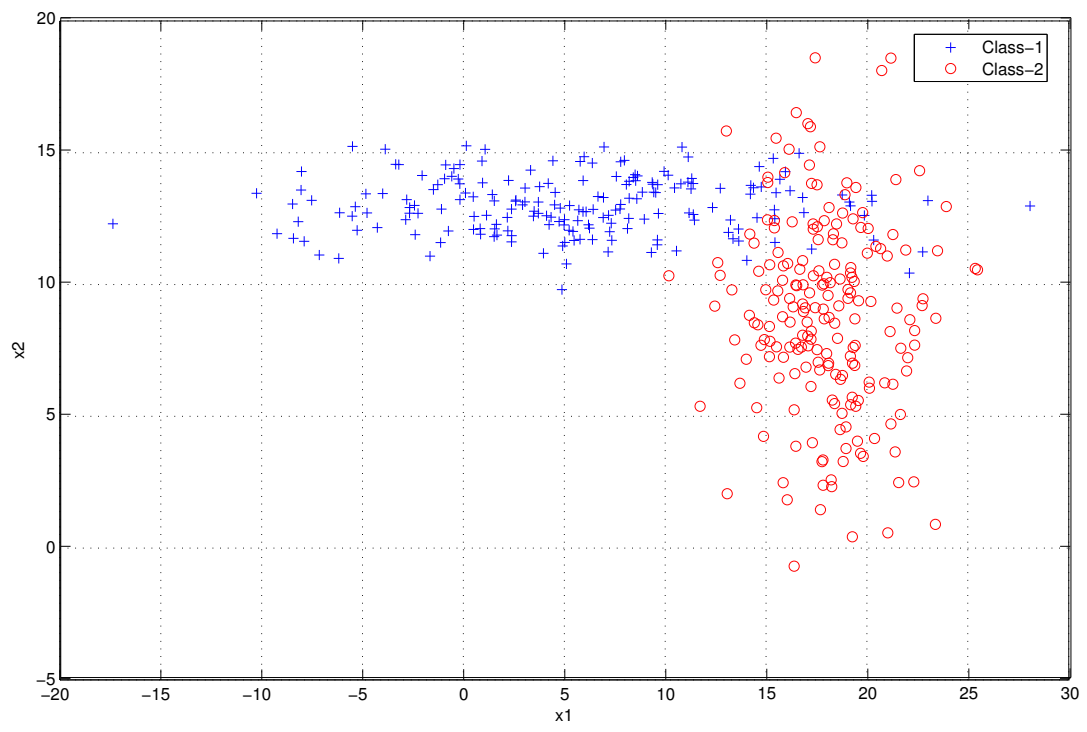

Figure 3.5: 2 class, 2 feature problem - Non linearly separable data

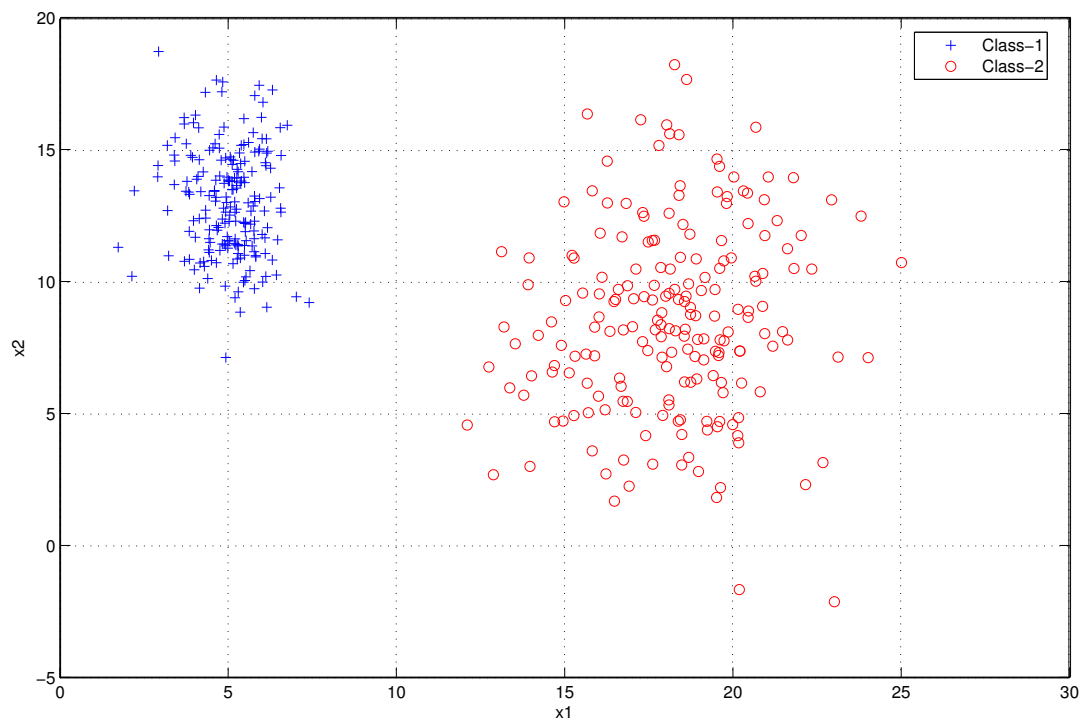

Figure 3.6: 2 class, 2 feature problem - Non linearly separable data becomes linearly separable after applying $2 \nu$-SVM 
moval, histogram equalization, entropy equalization, homomorphic filtering, and background subtraction. If $I$ is the original iris image, the set of enhancement functions can be expressed as,

$$
\begin{gathered}
I_{1}=f_{\text {noise }}(I) \\
I_{2}=f_{\text {blur }}(I) \\
I_{3}=f_{\text {focus }}(I) \\
I_{4}=f_{\text {histogram }}(I) \\
I_{5}=f_{\text {entropy }}(I) \\
I_{6}=f_{\text {filter }}(I) \\
I_{7}=f_{\text {background }}(I)
\end{gathered}
$$

where $I$ is the input training image, $f_{\text {noise }}$ is the algorithm for noise removal [49], $f_{\text {blur }}$ is the algorithm for blur removal [37], $f_{\text {focus }}$ is the algorithm for adjusting the focus of the image [61], $f_{\text {histogram }}$ is the histogram equalization function [32], $f_{\text {entropy }}$ is the entropy filter [38], $f_{\text {filter }}$ is the homomorphic filter [32] for contrast enhancement and $f_{\text {background }}$ is the background subtraction process [46]. $I_{1}, I_{2}, I_{3}, I_{4}, I_{5}, I_{6}$, and $I_{7}$ are the images obtained when the above enhancement operations are applied to the original iris image. In this research work, we have used the above referenced algorithms for noise removal, defocus, deblur, background subtraction and other enhancement algorithms. It is not necessary to 
use only these algorithms. Any algorithm which performs a given function well can be used or additional functions can be easily added.

\subsubsection{Image Fusion using 2 $\nu$-Support Vector Machine}

An intelligent learning algorithm based on $2 \nu$ - SVM is used to train and classify the pixel quality from corresponding locations of the preprocessed iris images. This knowledge is used for fusion algorithm that combines the good quality pixels from each of the transformed and the original iris image. The fusion algorithm uses $2 \nu$-SVM [9] as described in Section 3.2.1. The fusion algorithm is divided into two parts, $2 \nu$-SVM training, and $2 \nu$-SVM classification and enhancement.

\section{2 $\nu$-SVM Training}

$2 \nu$-SVM is trained to classify the input pixels of the iris image as good or bad. For this, few input and preprocessed iris images are labeled as good or bad and these labeled iris images are used for training the $2 \nu$-SVM. The training algorithm is defined as follows:

1. The training iris images are decomposed to $l$ levels by the Discrete Wavelet Transform. The $3 l$ detail subbands of each image contains the edge features and thus these bands are used for training.

2. The activity level of the wavelet coefficients for each detail subband is computed. The activity level is computed over a small window by treating each coefficient separately.

3. The $2 \nu$-SVM is trained using labeled iris image to determine the quality of every wavelet coefficient. For training, the activity levels computed in the previous step are given as input to the $2 \nu$-SVM.

4. The output of the training is a label $G$ or 1 if the coefficient is good and a label $B$ or 0 if the coefficient is bad. 


\section{2 $\nu$-SVM Classification and Enhancement}

The trained $2 \nu$-SVM is used to classify the pixels from the input image and to generate a new feature-rich iris image. The algorithm is described as follows:

1. The processed iris images generated in Section 3.2.2 along with the original image are decomposed to $l$ levels using the DWT.

2. $2 \nu$-SVM classifier is then used to classify the coefficients of the input bands as good or bad. A decision matrix Decision is generated which stores the quality of each coefficient in terms of $G$ and $B$. At any position $(i, j)$ if the SVM output $O(i, j)$ is positive then that coefficient is labeled as $G$ (Good) otherwise it is labeled as $B$ (Bad).

$$
\operatorname{Decision}(i, j)=\left\{\begin{array}{lll}
G, & \text { if } & O(i, j)>0 \\
B, & \text { if } & O(i, j)<0
\end{array}\right.
$$

3. This operation is performed on all eight images and the decision matrix is created corresponding to every image.

4. At every location $(x, y)$, the average of every coefficient whose label is $G$ is obtained and the coefficients with label $B$ is discarded. In this manner, $3 l$ fused detail subbands and one fused approximation band is generated.

5. Feature-rich iris images are further generated by applying inverse DWT on the fused coefficients.

Iris images are enhanced using proposed iris enhancement algorithm and these enhanced iris images are used for the recognition process. Figure 3.7 shows the process of iris image enhancement. 


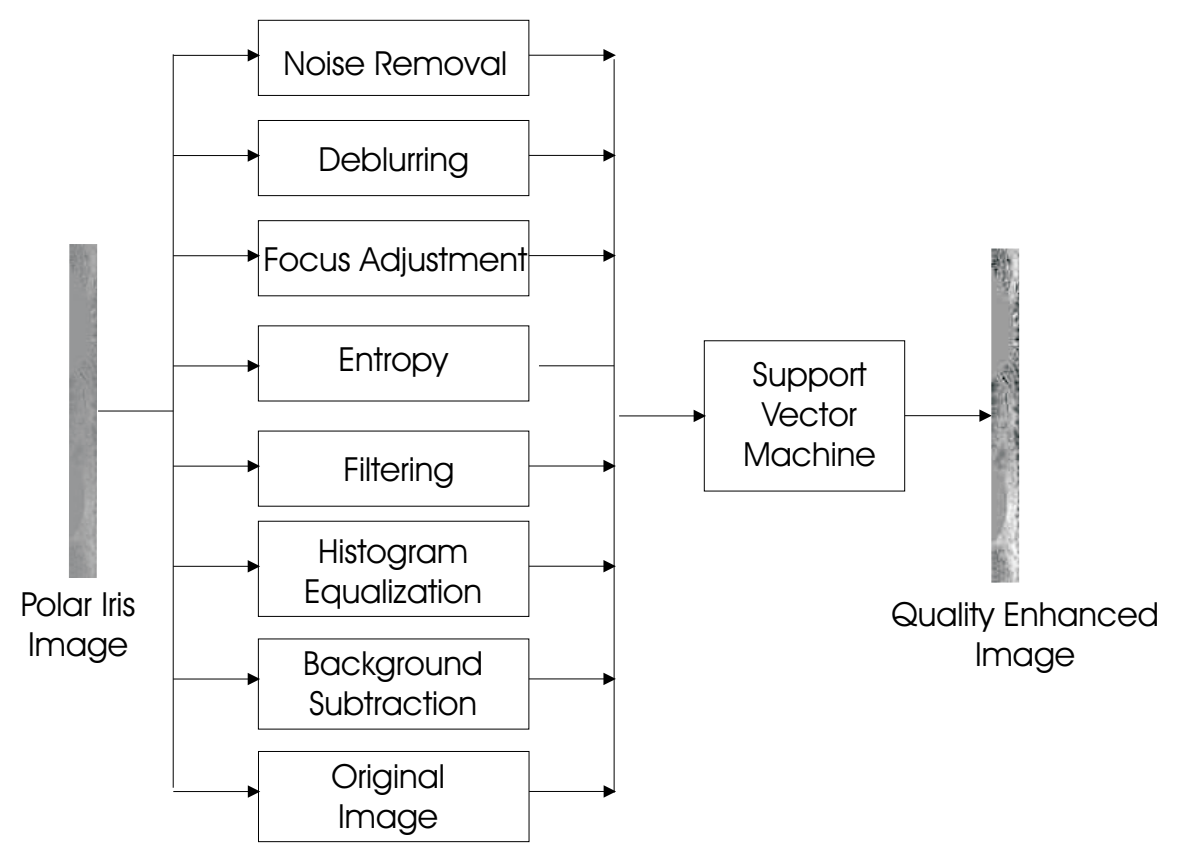

Figure 3.7: Iris image enhancement 


\section{Chapter 4}

\section{Iris Feature Extraction and Matching}

This chapter describes the extraction of textural and topological features from the preprocessed image using 1D log polar Gabor and Euler numbers respectively. The template generated by encoding textural features is called iris template and the template generated from topological features is called the Euler Code.

\subsection{Iris Texture Template Generation}

We propose the use of log polar form of 1D Gabor wavelet for iris texture template generation. Like Gabor wavelets, log polar Gabor filters are based on polar coordinates but unlike the frequency dependence on a linear graduation, the dependency is realized by a logarithmic frequency scale. Therefore, the functional form of 1D log polar Gabor wavelet is given by:

$$
G l p_{r_{0} \theta_{0}}(\theta)=\exp \left[-2 \pi^{2} \sigma^{2}\left[\left\{\ln \left(\frac{r-r_{0}}{f}\right)\right\}^{2} \tau^{2}+\left\{2 \ln \left(f_{0} \sin \left(\theta-\theta_{0}\right)\right)\right\}^{2}\right]\right]
$$

where $(r, \theta)$ are the polar co-ordinates, $r_{0}$ and $\theta_{0}$ are the initial values, $f$ is the center frequency of the filter and $f_{0}$ is the parameter which controls the bandwidth of the filter. $\sigma$ and $\tau$ are defined as follows: 


$$
\begin{gathered}
\sigma=\frac{1}{\pi \ln \left(r_{0}\right) \sin \left(\pi / \theta_{0}\right)} \sqrt{\frac{\ln 2}{2}} \\
\tau=\frac{2 \ln \left(r_{0}\right) \sin \left(\pi / \theta_{0}\right)}{\ln 2} \sqrt{\frac{\ln 2}{2}}
\end{gathered}
$$

In contrast to Gabor wavelets which are symmetric with respect to their principal axis, log polar Gabor filters show a translation of maximum from the center of gravity in the direction of a lower frequency and flattening of the high frequency part. The most important feature of the proposed filter is that it is rotation and scale invariant. Also log polar Gabor functions, having extended tails, encodes natural images more efficiently than Gabor functions. This is because Gabor function over-represents the low frequency components and under-represents the high frequency components in any encoding process [25]. Figure 4.1 shows the plot of 1D log polar Gabor transfer function.

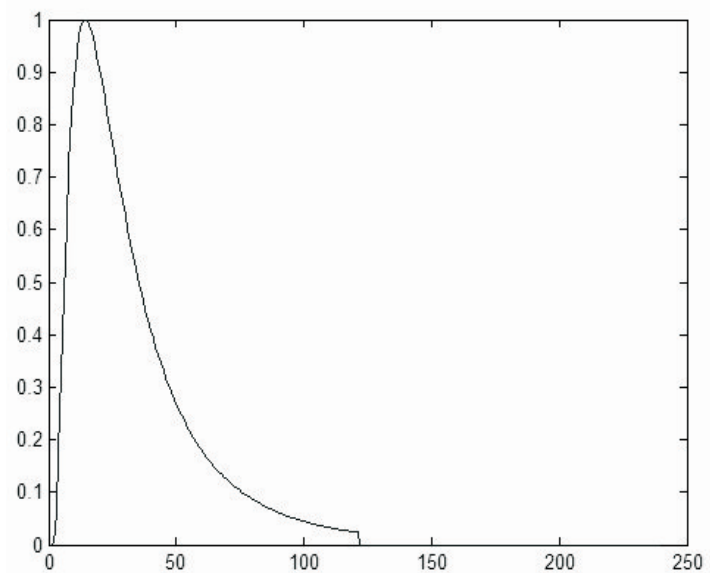

(a)

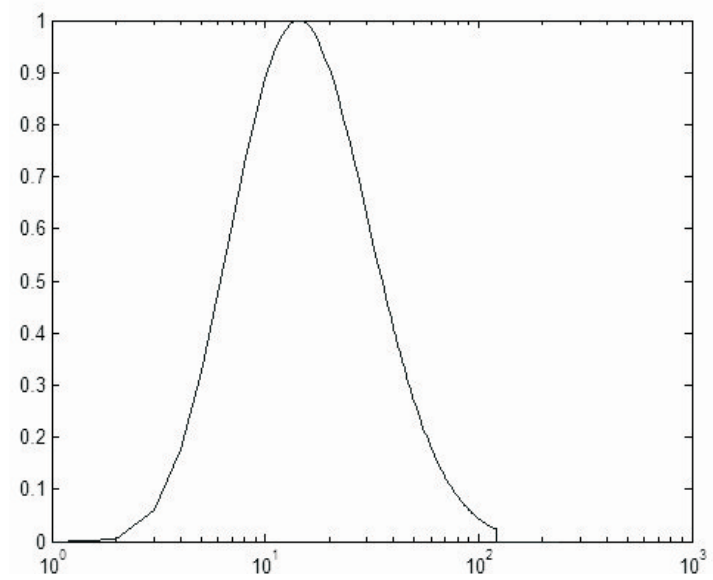

(b)

Figure 4.1: 1D log polar Gabor transfer function (a) Linear (b) Logarithmic

To generate an iris template from the proposed 1D log polar Gabor wavelet, the 2D normalized pattern, i.e. the quality enhanced polar iris image is decomposed into a number of $1 \mathrm{D}$ signals where each row corresponds to a circular ring on the iris region. Angular 
direction rather than radial is used because maximum independence occurs in the angular direction. These 1D signals are then convolved with the 1D log polar Gabor wavelet in frequency domain. The values of the convolved iris image are complex in nature. Using these real and imaginary values, the phase information is extracted and encoded in a binary pattern. If the convolved iris image is $I_{g}(r, \theta)$, then the phase feature $P(r, \theta)$ is calculated using Equation 4.4,

$$
\begin{gathered}
P(r, \theta)=\tan ^{-1}\left(\frac{\operatorname{Im} I_{g}(r, \theta)}{\operatorname{Re} I_{g}(r, \theta)}\right) \\
I_{p}[r, \theta]=\left\{\begin{array}{lll}
{[1,1]} & \text { if } \quad 0^{0}<P(r, \theta) \leq 90^{\circ} \\
{[0,1]} & \text { if } & 90^{0}<P(r, \theta) \leq 180^{\circ} \\
{[0,0]} & \text { if } & 180^{0}<P(r, \theta) \leq 270^{\circ} \\
{[1,0]} & \text { if } & 270^{\circ}<P(r, \theta) \leq 360^{\circ}
\end{array}\right.
\end{gathered}
$$

where $I_{p}(r, \theta)$ is the binary iris template and the process is known as phase quantization. The iris template is a bitwise template containing a number of bits of information, and a noise mask which corresponds to noisy areas within the iris pattern. Figure 4.2 shows the iris template, its corresponding mask, and the convolved iris image. Figure 4.3 shows the complete iris code generation process.

\subsection{Euler Code Generation}

Convolution with the log polar Gabor wavelet gives the global textural characteristics of the iris image but does not extract the local features. Performance can be further improved if we also incorporate the topological features. For this, we used Euler numbers [32] which give the topological features of the iris. For a binary image, Euler number can be defined as the difference of number of connected components and the number of holes. Euler numbers are also invariant to rotation, translation, scaling and rubber sheet transformation of the image. Each pixel of the unwrapped iris can be represented as an 8-bit binary vec- 


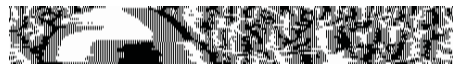

(a) Iris template
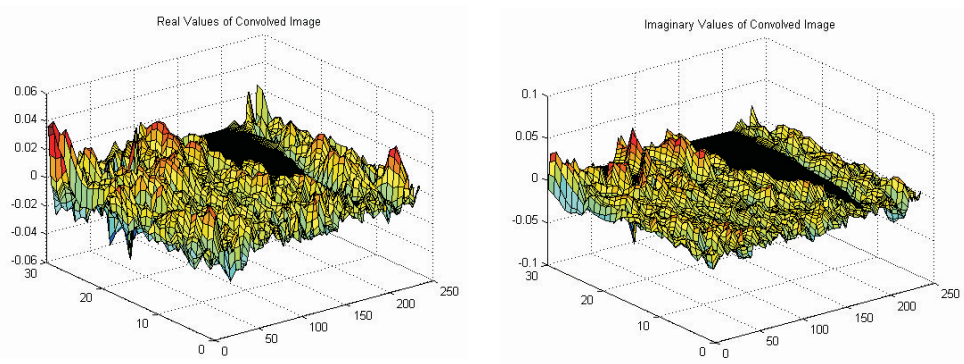

(c) and (d) Real and imaginary part of the convolved iris image

Figure 4.2: Iris template

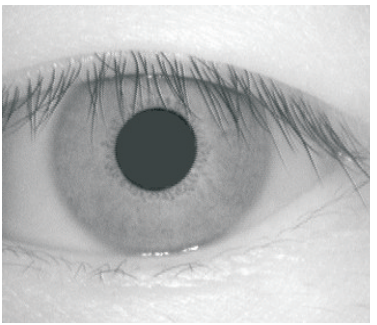

(a) Eye image

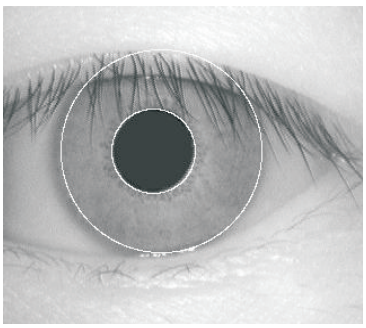

(b) Inner and outer boundaries

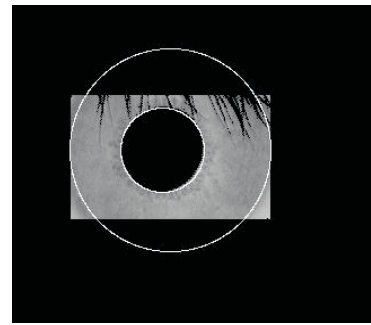

(c) Iris portion in the eye image

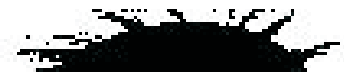

(d) Mask for the noise present in the iris image

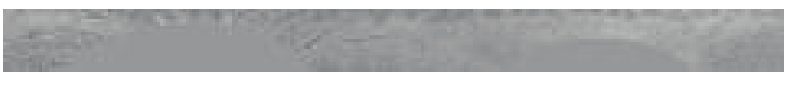

(e) Mask applied to the unwrapped iris image

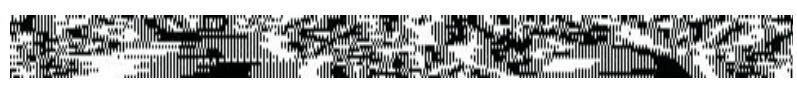

(f) Iris template

Figure 4.3: Iris template generation 
tor $\left\{b_{7}, b_{6}, b_{5}, b_{4}, b_{3}, b_{2}, b_{1}, b_{0}\right\}$, with each bit either ' 0 ' or ' 1 '. These bits form 8 planes with the binary values. Among these, four planes formed with the four Most Significant Bits $(M S B \mathrm{~s})$ represent the structural information of iris and the remaining four planes represent the brightness information and are random in nature. Brightness information is redundant for comparing the structure of two iris images. Thus 4-tuple Euler Code is formed with the four $M S B$ planes of the masked polar iris.

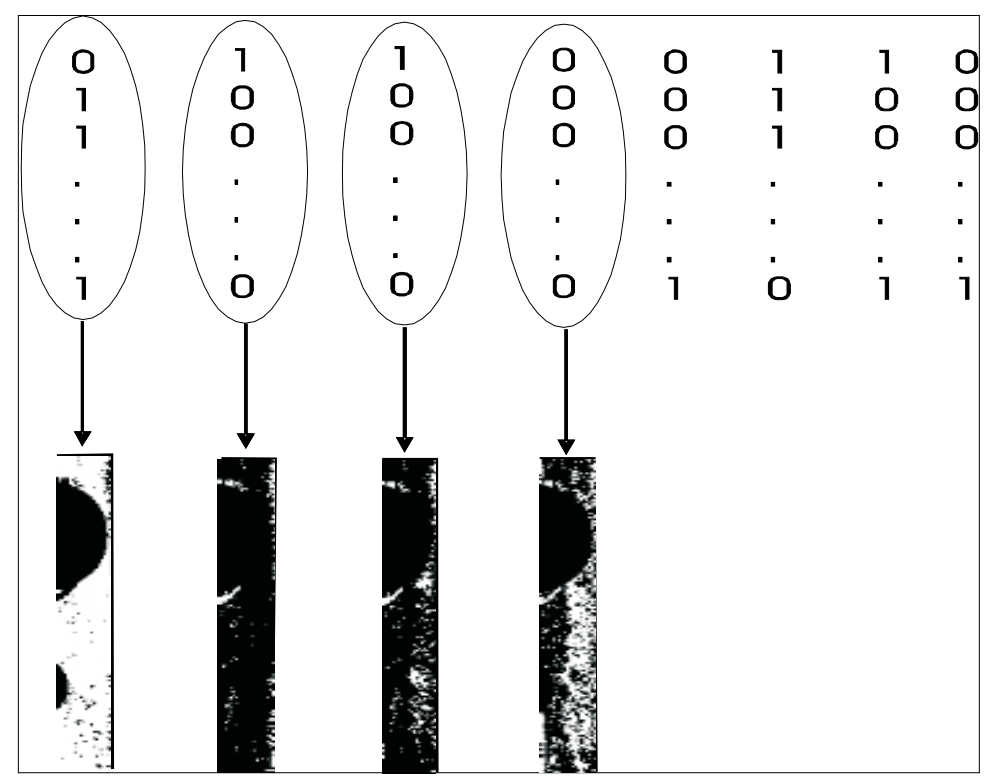

Figure 4.4: Generating four binary images from the masked polar image

For comparing two polar iris images using Euler Code, a common mask is generated using the algorithm in Section 3.1. This common mask is obtained by performing a bitwise-OR operation of the two individual masks and is applied to both the polar images. The 4-tuple Euler Code is generated from the masked polar iris images to be matched. Figure 4.5 shows the Euler Codes of a person at three different instances.

\subsection{Feature Matching}

To verify a person's identity, we match the query iris template with the stored template. Hamming Distance (HD) based matching algorithm [17] is used for matching the textural 


\section{EULER VECTOR}

$\begin{array}{lllll}\text { IMAGE } 1 & -72 & 64 & 140 & -365 \\ \text { IMAGE } 2 & -62 & 84 & 148 & -371 \\ \text { IMAGE 3 } & -63 & 75 & 145 & -368\end{array}$

Figure 4.5: Euler code

iris template and Mahalanobis distance (MD) based similarity matching is used for matching the Euler codes.

\subsubsection{Iris Code Matching}

For the two masked binary templates $A_{i}$ and $B_{i}, H D$ can be calculated as:

$$
H D=\frac{1}{N} \sum_{i=1}^{N} A_{i} \oplus B_{i}
$$

and

$$
M S_{I T}=1-H D
$$

where $N$ is the number of bits represented by each template and $\oplus$ is $\mathrm{XOR}$ operation. $H D$ gives the matching score $M S_{I T}$ for the iris template as given in Equation 4.6. For handling rotation, the templates are shifted left and right bit-wise and a number of $H D$ values are calculated from successive shifts [17]. The smallest value is used as the final $H D$ to calculate the matching score. This bit-wise shifting in the horizontal direction corresponds to rotation of the original iris region at an angle given by the angular resolution used. It is capable of taking into account the misalignments in the normalized iris pattern caused by rotational differences during imaging. 


\subsubsection{Euler Code Matching}

Mahalanobis distance based matching algorithm is used to match the two Euler codes. Mahalanobis distance between two points can be defined as,

$$
M D(\underline{x}, \underline{y})=\sqrt{(\underline{x}-\underline{y})^{t} S^{-1}(\underline{x}-\underline{y})}
$$

where, $\underline{x}$ and $\underline{y}$ are the two Euler codes to be matched, and $S$ is the positive definite covariance matrix of $\underline{x}$ and $\underline{y}$. Mahalanobis distance ensures that the features having high variance should not contribute to the distance. If Euler code has large variance, it increases the false reject rate. Applying the Mahalanobis distance metric for comparison thus avoids the increase in the false reject rate. This distance metric captures a very good similarity or dissimilarity between the two Euler codes.

$$
M S_{E C}=\frac{M D(\underline{x}, \underline{y})}{\log _{10} \max (M D)}
$$

As shown in Equation 4.9, the matching score of Euler code is the normalized Mahalanobis distance between the two codes where $\max (M D)$ is the maximum possible value of Mahalanobis distance between two Euler codes.

\subsection{Information Fusion}

Iris recognition algorithms have succeeded in achieving a low false acceptance rate but reducing the rejection rates is still a challenge. To make the iris recognition algorithms more practical and adaptable to diverse applications, false rejection rate needs to be reduced significantly. In this section, we propose three fusion algorithms to fuse the textural and the topological features of an iris image so as to reduce the false rejection rate while maintaining a low false acceptance rate. The three fusion algorithms are: 
1. Empirical Decision Fusion

2. SVM based Expert Fusion

3. SVM based Match Score Fusion

\subsubsection{Empirical Decision Fusion}

A decision fusion algorithm is proposed which is based on the empirical values obtained after exhaustive experiments. First, the template database is prepared using the strategy shown in Figure 4.6(a). For getting enrolled in the database at least $60 \%$ of the iris information should be present, i.e. noise in the image due to eyelids and eyelashes or the total area covered by the mask should be less than $40 \%$. Both the iris template and the Euler code of iris images are stored in the database. Database contains three instances of both the iris template and the Euler code.

For verifying the identity of an individual, we first check for the useful information present in the image. If more than $40 \%$ of the iris region is occluded by noise then according to this decision fusion approach, the iris image is discarded as it does not contain enough features for matching. If accepted from this quality check, the features are encoded from the unwrapped iris image and the iris templates and Euler codes are matched using the matching algorithms described in Section 4.3. Match scores obtained are then fused with the decision strategy described as follows:

1. If the three matching scores $M S_{I T}$ obtained by matching the input iris template with the three database images are less than a threshold, thresh $h_{H D 1}$, then the person is accepted.

2. If the three matching scores $M S_{I T}$, obtained by matching the iris templates are greater than a threshold, thresh ${ }_{H D 2}$, then the person is rejected.

3. If the textural features are not able to give a perfect accept or perfect reject decision, then we additionally use the results obtained from Euler code. Thus, if both the above 
conditions are not satisfied then,

(a) The matching scores are sorted in descending order and the following equation is applied to get a new combined matching score $M S_{\text {new }}$

$$
M S_{I T-n e w}=\frac{s 1 * M S_{I T(\max )}+s 2 * M S_{I T(\operatorname{mid})}+s 3 * M S_{I T(\min )}}{s 1+s 2+s 3}
$$

where $s 1, s 2$, and $s 3$ are the weights assigned to the three matching scores. These values are derived empirically such that $s 1>s 2>s 3$. This equation thus assigns more weight to the matching score by which a user is accepted and less to the matching score by which a user is rejected.

(b) Average value of the three matching scores obtained from the Euler code matching is calculated $M S_{E C-a v g}$,

$$
M S_{E C-a v g}=\frac{M S_{E C 1}+M S_{E C 2}+M S_{E C 3}}{3}
$$

where, $M S_{E C 1}, M S_{E C 2}$, and $M S_{E C 3}$ are the three matching scores.

(c) Depending on the decision rule in Equation 4.11, the person is said to be accepted $(A)$ or rejected $(R)$,

$$
\text { Decision }= \begin{cases}A & \text { if } M S_{\text {new }} \leq T_{H D-\text { new }} \text { and } M S_{E C-a v g} \leq T_{E C-\text { avg }} \\ R & \text { otherwise }\end{cases}
$$

We also varied the number of training samples in the database from one to three and found that using the decision fusion algorithm, the best performance is achieved with three training samples. The set of thresholds used to get the best performance are given below:

1. thresh $_{H D 1}=0.36$, and thresh ${ }_{H D 2}=0.40$ 
2. $s 1=0.3, s 2=0.3$, and $s 3=0.1$

3. $T_{H D-n e w}=0.38$ and $T_{E C-a v g}=2$

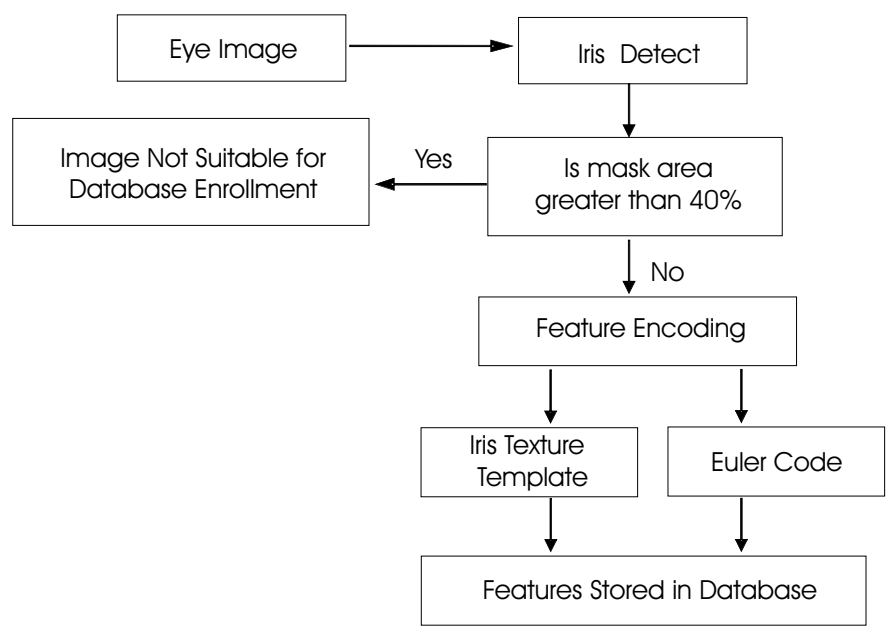

(a)

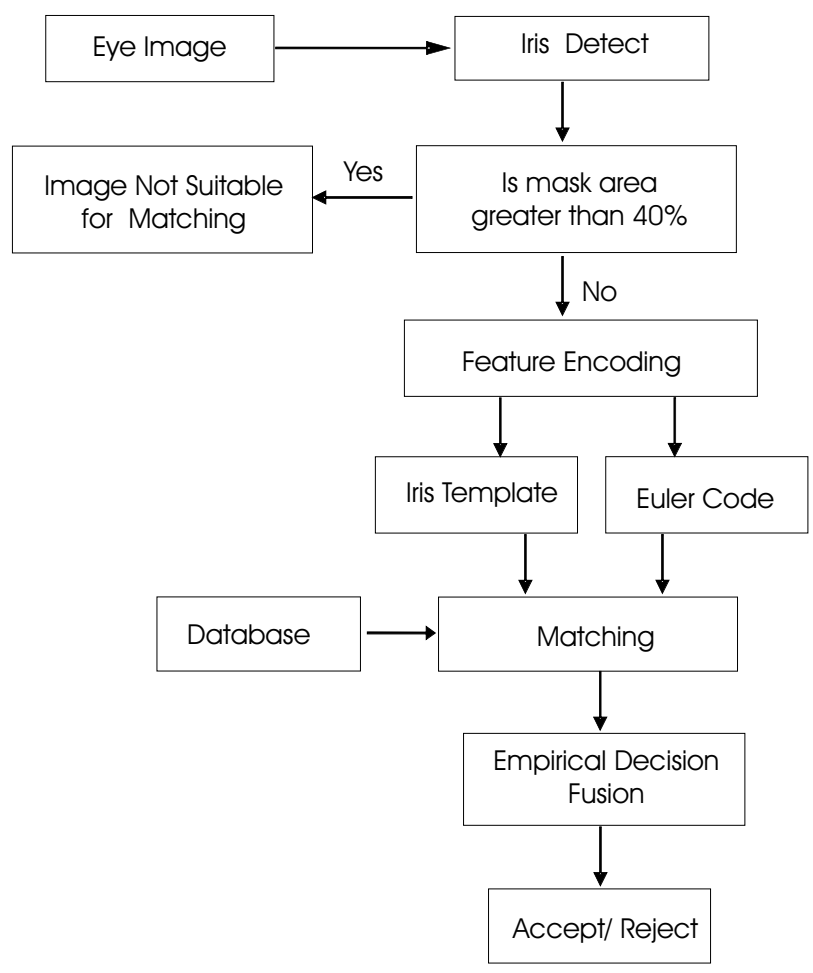

(b)

Figure 4.6: (a) Database enrollment and (b) Matching strategy 


\subsubsection{SVM based Decision Fusion}

In a multiple expert system, there are $n$ experts and one administrator. Expert provides their opinions about the claims of a candidate and administrator makes the decision based on the opinions. In expert fusion, the administrator makes the decision based on the expert opinion and the confidence level of the expert. For example, one expert is $10 \%$ confident that a genuine user should be rejected using the iris template and another expert is $99 \%$ sure that the same user should be accepted using Euler code then the administrator decides to accept the user. In another example of expert fusion, a genuine user is rejected by Euler code with $45 \%$ confidence and iris template expert with $80 \%$ confidence says that the person should be accepted. In this case also, the administrator should accept the candidate. In the cases when any impostor tries to impersonate someone's identity Euler code based expert may accept the candidate with $60 \%$ confidence but the iris template based expert may reject with $98 \%$ confidence. In such cases, the administrator rejects the candidate because the iris code based expert is more confident of the decision. Based on the above three examples, we can come up with three challenges for expert fusion,

- Who are the experts and how reliable are the experts?

- How does an administrator make decisions based on opinions rendered by different experts?

- How do we normalize the data?

In [5], [24], and [72], detailed experimental evaluations of multiple expert fusion algorithms are presented. Most of the research in expert fusion is based on statistical evaluation or Bayesian approach. Here we present an approach of expert fusion based on Support Vector Machine.

Let the two feature sets be $f_{1}$ (iris code) and $f_{2}$ (Euler code), which are used by the experts $e_{1}$ and $e_{2}$ respectively. Let $g_{1}$ and $g_{2}$ be the confidence of experts $e_{1}$ and $e_{2}$, 


$$
g_{1}=\frac{1}{\sqrt{S R_{1}}}, g_{2}=\frac{1}{\sqrt{S R_{2}}}
$$

where $S R_{1}$ and $S R_{2}$ are the success rates defined as,

$$
S R=1-\left(\frac{F A+F R}{N}\right)
$$

where, $F A$ is the number of false acceptance cases, $F R$ is the number of false rejection cases and $N$ is the number of attempts. In the training phase, SVM for both the experts are trained using labeled positive and negative classes as described in Section 3.2.1. $g_{1}$ and $g_{2}$ are calculated using Equation 4.13 and expert training data.

In the recognition phase, when two test features (iris code and Euler code) are given to experts $e_{1}$ and $e_{2}$ respectively, the experts try to find the decision boundary by minimizing the following equation from Section 3.2.1,

$$
\min \left(\frac{1}{2}\left\|{\sqrt{g_{i}^{2} \omega_{i}^{2}}}^{2}\right\|-\sum_{j} C_{j}\left(\nu \rho-\varphi_{j}\right)\right)
$$

subject to,

$$
y_{j}\left(w_{i} \varphi(x)+b\right) \geq \rho-\varphi_{j}, \quad \phi_{j} \geq 0, \quad \forall j \text { and } i=1,2
$$

If the margin from the decision boundary for expert $e_{1}$ is greater than $e_{2}$, then administrator accepts the decision of $e_{1}$, otherwise the administrator accepts the decision of $e_{2}$. Here, an adaptive scheme for $g_{i}$ can also be implemented. Figure 4.7 shows the steps involved in the expert fusion algorithm. 


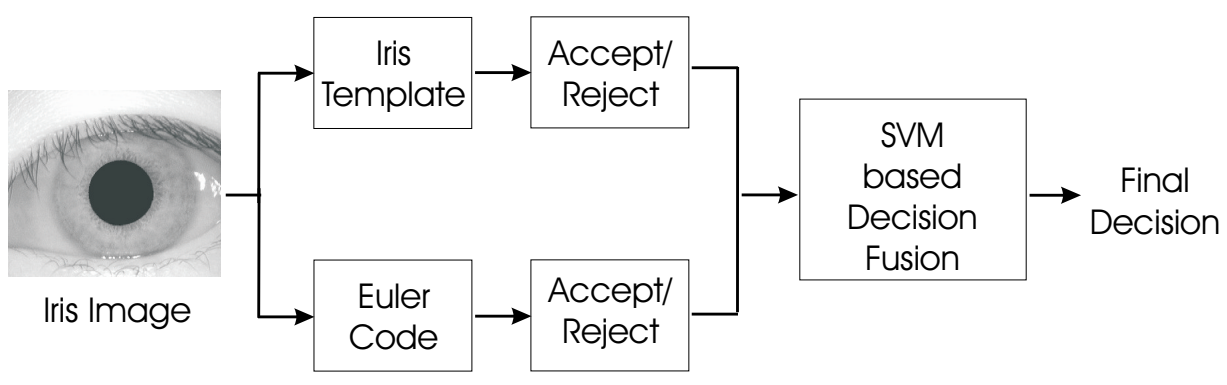

Figure 4.7: Steps involved in expert fusion

\subsubsection{SVM based Match Score Fusion}

In [39], [48], it has been suggested that the fusion of match scores of two or more classifiers gives better performance compared to a single classifier. In general, match score fusion is performed using sum rule, product rule or other statistical rules. Recently in [1], a SVM based match score fusion algorithm is proposed to fuse the match scores of fingerprint and signature. In this section, we describe a novel SVM learning based match score fusion algorithm which can be used to fuse the two match scores from iris template and Euler code.

Let the training set be $Z=\left(x_{i}, y_{i}\right)$ where $i=1, \ldots, N$, with $N$ being the number of multimodal scores used for training and $y_{i} \epsilon(1,-1)$, where 1 represents the genuine class and -1 represents the impostor class. The SVM is trained using these labeled training data. The training data is mapped in a higher dimension feature space such that $Z \rightarrow \varphi(Z)$ where $\varphi($.) is the mapping function. Optimal hyperplane which separates the data into two different classes in the higher dimensional feature space can be obtained as the solution of Equation 3.5 in Chapter 3.

In the testing phase, fused score $f_{t}$ of a multimodal test pattern $x_{t}$ is defined as (from Chapter 3)

$$
f_{t}=f\left(x_{t}\right)=\omega \varphi\left(x_{t}\right)+b
$$

Solution of this equation is the signed distance of $x_{t}$ to the separating hyperplane given 
by the SVM. Finally, an accept or reject decision is made on the test pattern $x_{t}$ as follows:

$$
\operatorname{result}\left(x_{t}\right)=\left\{\begin{array}{lll}
\text { accept, } & \text { if } & \text { output of } S V M>0 \\
\text { reject, } & \text { if } & \text { output of } S V M<0
\end{array}\right.
$$

Match score fusion algorithm described above is the global training and decision scheme. However, we can also design the local and/or adaptive learning and decision schemes based on the above concept. Figure 4.8 represents steps involved in the match score level fusion using SVM. Using the proposed SVM learning based match score fusion algorithm, the match scores of iris code and Euler code are fused to get a better classification result. Here the learning algorithm performs the classification between genuine and imposter matching scores.

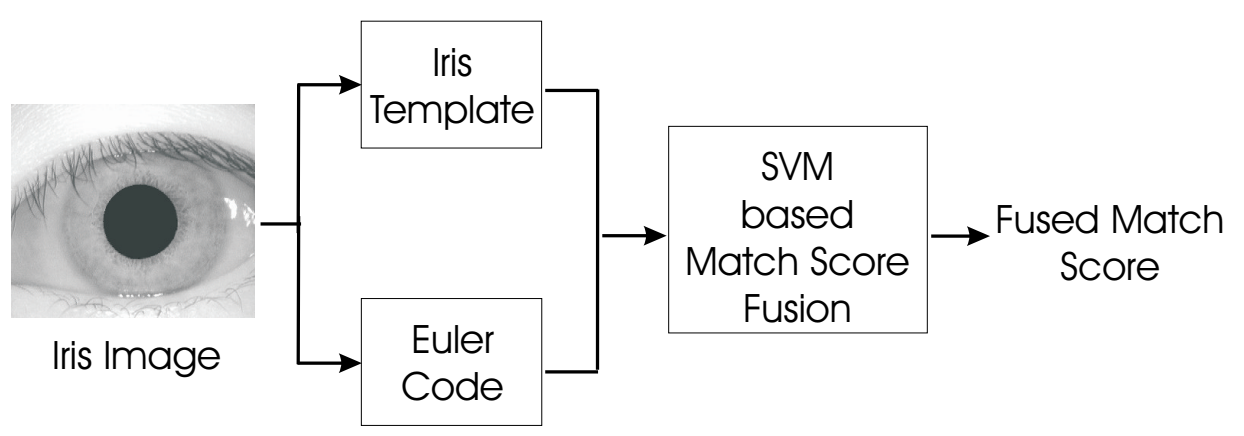

Figure 4.8: Steps involved in match score fusion 


\section{Chapter 5}

\section{Experimental Results}

The proposed iris image enhancement and recognition algorithms are validated by computing the matching performance of iris images. Experiments are performed using four iris image databases, CASIA iris database [80], Miles Research Database [82], UBIRIS [56] and UPOL iris database [22]. These four iris databases are prepared from different countries, in different imaging environments, and contain iris images from individuals belonging to different ethnicity and geographical locations. This provides us with an opportunity to test and validate the performance of our algorithms on a diverse database.

\subsection{Experimental Results of Proposed Algorithms}

In this section, results of the proposed segmentation, preprocessing, feature extraction and matching algorithms are presented. Sample results of iris segmentation, preprocessing and feature template generation using 1D log polar Gabor wavelet are shown in Figure 5.1 and Figure 5.2. In these figures, (a) is the input eye image, (b) is the detected inner and outer boundaries of iris, (c) shows the detected iris region, (d) is the mask for the noise present in the image, (e) is the segmented iris image in polar coordinates (f) is the enhanced quality iris image and $(\mathrm{g})$ is the iris template generated from 1D log polar Gabor.

Figure 5.3 shows the result demonstrating the effectiveness of the proposed iris segmentation and preprocessing algorithm. In this figure, a major portion of the iris image is covered 


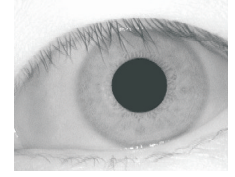

(a) Eye image

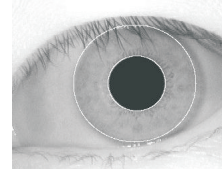

(b) Inner and outer boundaries

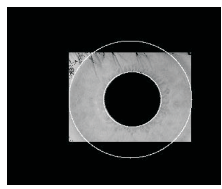

(c) Iris segmentation

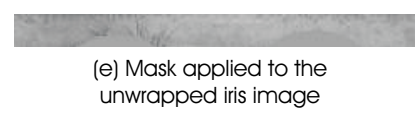

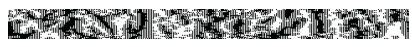

(g) Iris template

Figure 5.1: Iris template generation

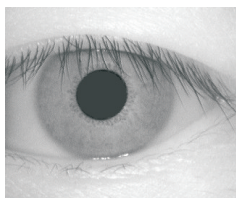

(a) Eye image

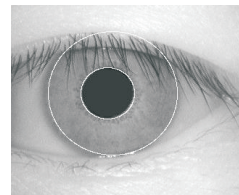

(b) Inner and outer boundaries

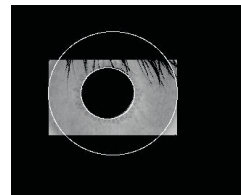

(c) Iris segmentation

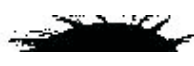

(d) Mask for the noise present in the image

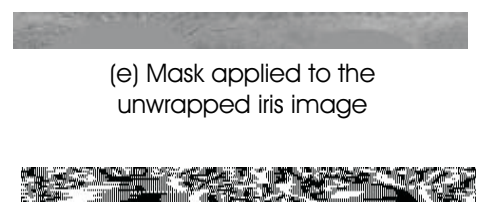

(g) Iris template

(f) Enhanced iris image

Figure 5.2: Iris template generation with eyelids

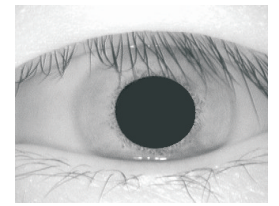

(a) Eye image

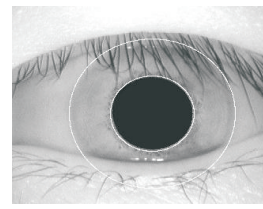

(b) Inner and outer boundaries

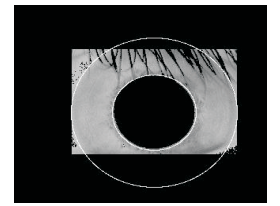

(c) Iris segmentation

\section{6 wh.}

(d) Mask for the noise present in the image

(e) Mask applied to the unwrapped iris image

Figure 5.3: Elliptical iris segmentation 

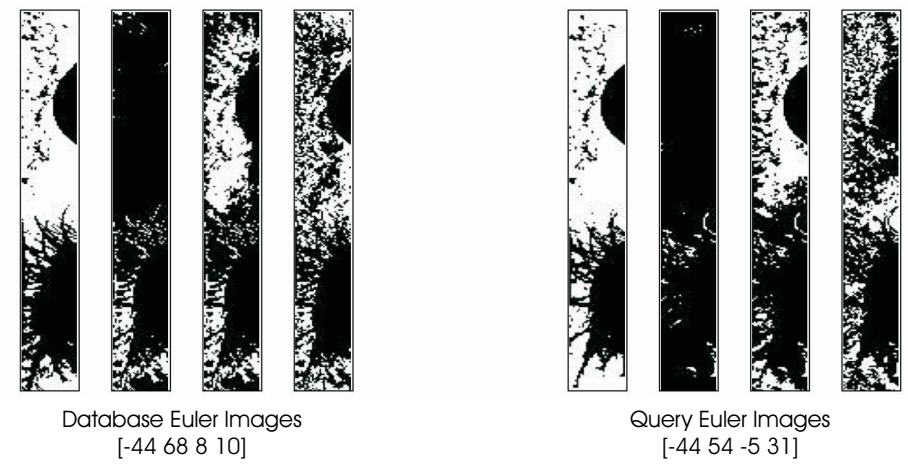

Figure 5.4: Matching two Euler codes

with eyelids and eyelashes and even the shape of the iris is ellipse like. The proposed segmentation algorithm effectively extracts the iris region from the eye image without assuming the boundary to be circular.

Figure 5.4 shows an example of Euler code generation. In this example, first set is the database Euler image and other set is the query Euler image. The two Euler codes are matched using the Mahalanobis distance.

Figure 5.5 shows ROC plot for recognition with quality enhanced iris images from the CASIA iris database [80]. This ROC plot shows that the proposed SVM based match score fusion algorithm performs best followed by empirical decision fusion algorithm and the SVM decision fusion algorithm. However, the performance of SVM decision fusion algorithm is very close to the performance of textural feature based iris recognition algorithm. This is because, in most of the cases, decision boundary of textural features is better than the topological features. The performance increases with empirical decision fusion because the decision fusion algorithm reduces the false rejection rate.

ROC plot for the experiments performed using the Miles database [82] is shown in Figure 5.6. This experiment also shows that the SVM based match score fusion performs the best followed by the empirical decision fusion and SVM decision fusion. Since the visual quality of the iris images in this database is very good, it can be assumed that the results should be near perfect which is reflected in this experiment.

The same set of experiments is performed using the UBIRIS database [56]. This database 


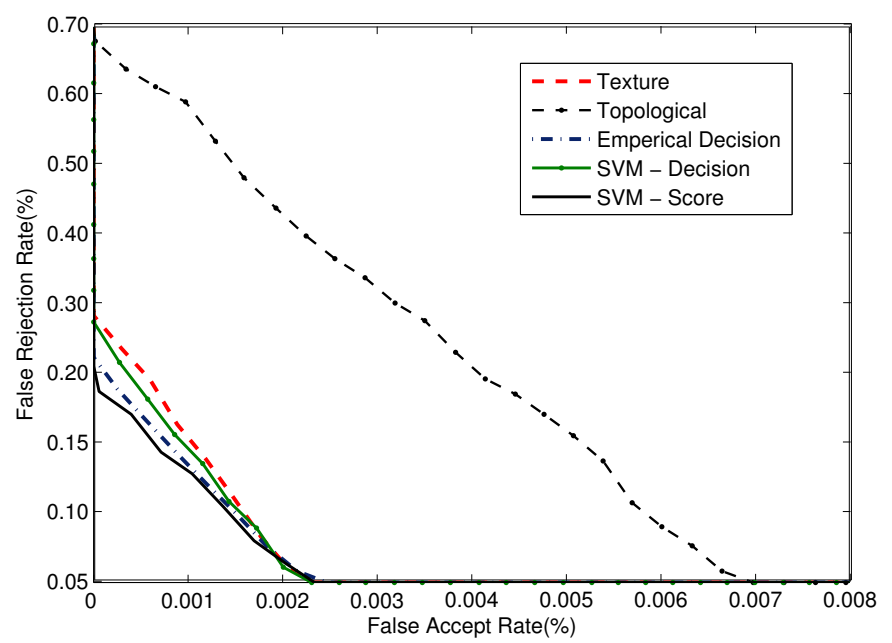

Figure 5.5: ROC plot showing the performance of proposed algorithms on the CASIA iris database [80]

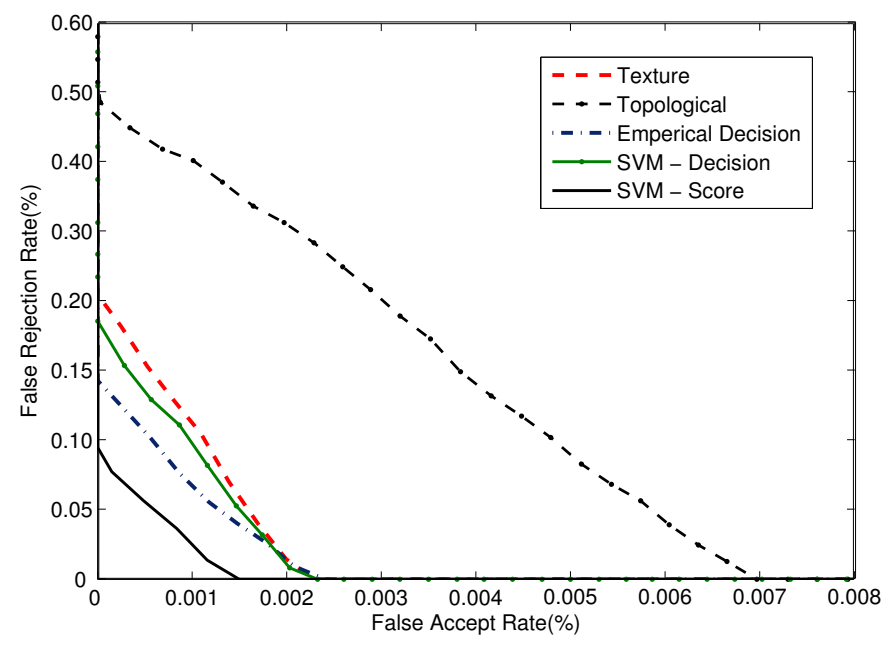

Figure 5.6: ROC plot showing the performance of proposed algorithms on Miles iris database $[82]$ 


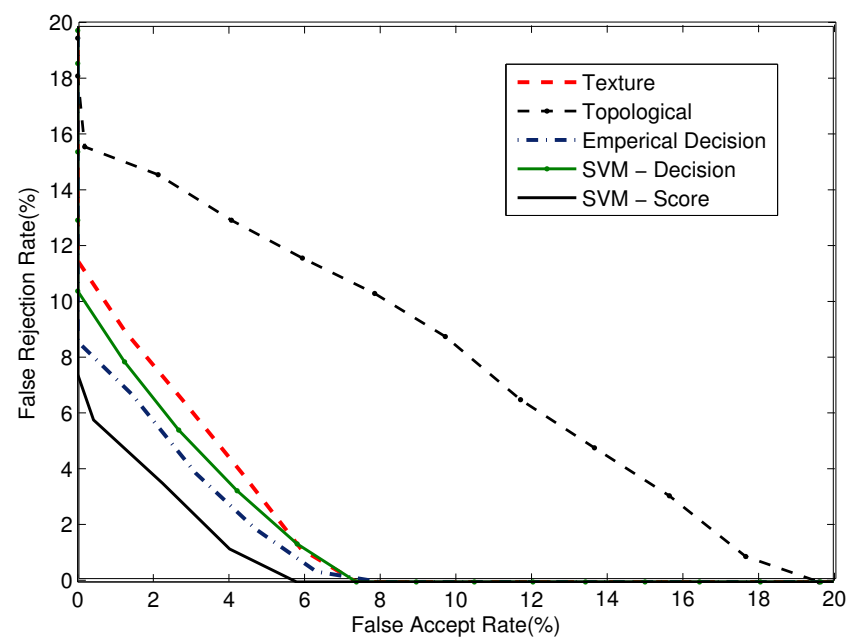

Figure 5.7: ROC plot showing the performance of proposed algorithms on UBIRIS iris database [56]
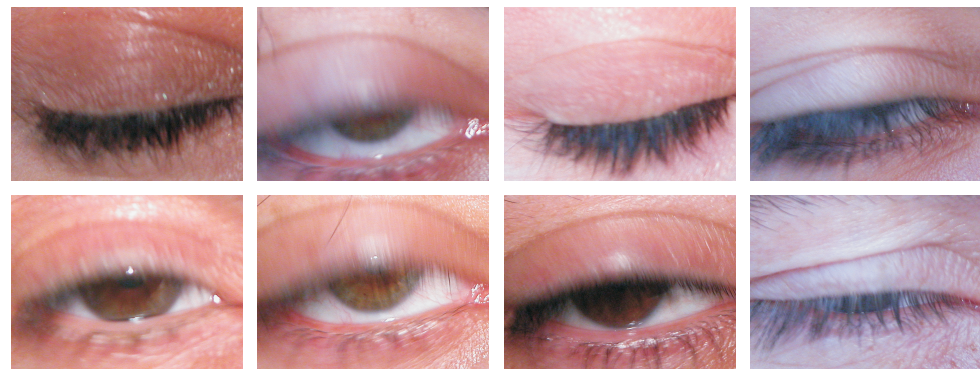

Figure 5.8: Sample iris images from UBIRIS database [56] on which the proposed algorithms fail to perform

contains images captured under different conditions and provides a challenging scenario for iris recognition. The images contain several noise factors such as motion blur, off angle, gaze direction, diffusion, and other real world problems, which give an opportunity to evaluate the robustness of the proposed algorithms. Figure 5.7 shows the ROC plot of this set of experiments. In this experiment, we found that the best performance is achieved using SVM based match score fusion with $7.35 \% \mathrm{FRR}$ at $0 \% \mathrm{FAR}$. This high rate of false rejection is due to the cases where the iris is not opened properly. Examples of such cases is shown in Figure 5.8.

We have also performed the experiments on UPOL iris database [22] and the ROC plot 


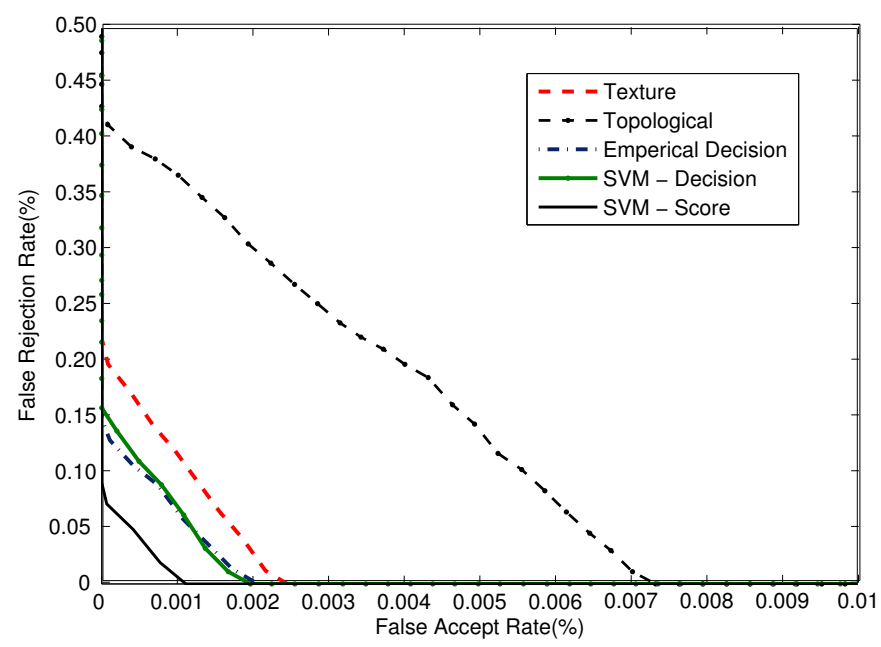

Figure 5.9: ROC plot showing the performance of proposed algorithms on UPOL iris database $[22]$

for this experiment is shown in Figure 5.9. Iris images in this database are of high quality and the noise present is minimal. So, the iris images in this database can be easily segmented. Results show that the SVM based match score fusion algorithm performs the best in this experiment with $0.08 \% \mathrm{FRR}$ at $0 \% \mathrm{FAR}$.

We summarized the experimental results on the four databases in Table 5.1. In this table, it can be seen that using the fusion algorithms, false rejection rates can be significantly reduced. However, the rejection rate cannot be reduced if a closed eye image or an eye image with very less information is captured.

\subsection{Performance of Iris Quality Enhancement Algo- rithm}

Next, the performance of the proposed image quality enhancement algorithm was evaluated. In this experiment, we chose four algorithms: textural features extracted using 1D log polar Gabor wavelet (iris code), Euler code based topological features, SVM based match score fusion of the textural and topological features, and pseudo implementation of Daug- 
Table 5.1: Summarizing the performance of proposed algorithm on the four iris databases

\begin{tabular}{|c|c|c|}
\hline \hline Database & Algorithm & FRR at 0\% FAR \\
\hline \multirow{4}{*}{ CASIA [80] } & Texture & $0.27 \%$ \\
\cline { 2 - 3 } & Topological & $0.68 \%$ \\
\cline { 2 - 3 } & Empirical Decision Fusion & $0.22 \%$ \\
\cline { 2 - 3 } & SVM Decision Fusion & $0.26 \%$ \\
\cline { 2 - 3 } & SVM Match Score Fusion & $0.20 \%$ \\
\hline \multirow{5}{*}{ Miles [82] } & Texture & $0.20 \%$ \\
\cline { 2 - 3 } & Topological & $0.49 \%$ \\
\cline { 2 - 3 } & Empirical Decision Fusion & $0.14 \%$ \\
\cline { 2 - 3 } & SVM Decision Fusion & $0.18 \%$ \\
\cline { 2 - 3 } & SVM Match Score Fusion & $0.09 \%$ \\
\hline \multirow{5}{*}{ UBIRIS [56] } & Texture & $11.72 \%$ \\
\cline { 2 - 3 } & Topological & $17.83 \%$ \\
\cline { 2 - 3 } & Empirical Decision Fusion & $8.79 \%$ \\
\cline { 2 - 3 } & SVM Decision Fusion & $10.47 \%$ \\
\cline { 2 - 3 } & SVM Match Score Fusion & $7.35 \%$ \\
\hline \multirow{5}{*}{ UPOL [22] } & Texture & $0.21 \%$ \\
\cline { 2 - 3 } & Topological & $0.43 \%$ \\
\cline { 2 - 3 } & Empirical Decision Fusion & $0.15 \%$ \\
\cline { 2 - 3 } & SVM Decision Fusion & $0.16 \%$ \\
\cline { 2 - 3 } & SVM Match Score Fusion & $0.08 \%$ \\
\hline \hline
\end{tabular}


Table 5.2: Performance of iris recognition algorithms with and without enhancement (Number of training image $=1$ )

\begin{tabular}{|c|c|c|}
\hline \hline $\begin{array}{c}\text { Existing Iris Recognition } \\
\text { Algorithms }\end{array}$ & $\begin{array}{c}\text { FRR without Iris Image } \\
\text { Quality Enhancement }\end{array}$ & $\begin{array}{c}\text { FRR with Iris Image } \\
\text { Quality Enhancement }\end{array}$ \\
\hline Texture - Iris Code & $0.57 \%$ & $0.27 \%$ \\
\hline Topological - Euler & $1.79 \%$ & $0.68 \%$ \\
\hline SVM based Match Score Fusion & $0.38 \%$ & $0.20 \%$ \\
\hline Daugman's Implementation & $0.37 \%$ & $0.18 \%$ \\
\hline
\end{tabular}

man's algorithm [17]. Matching performance is first calculated using original images from the database and then with the quality enhanced images. Matching performance is represented in terms of the false rejection rate (FRR) at $0 \%$ false acceptance rate (FAR). Table 5.2 shows that on the CASIA database, the proposed iris enhancement algorithm reduces the false rejection rate of iris recognition algorithms by approximately $50 \%$.

We can further reduce the false rejection rate by increasing the number of training images. In some cases, occlusion due to eyelids, eyelashes, spot light and noise present reduces the features available for matching. For such cases, increasing the number of training images increases the amount of information present in the images and thus reduces the false reject rate. Figure 5.10 and Figure 5.11 show the results with increasing the number of training images on the CASIA database. FRR decreases for both the Daugman's algorithm [17] and the proposed SVM based match score fusion algorithm. These two figures also show that if the training images are enhanced using the proposed quality enhancement algorithm then the FRR is reduced to $0.067 \%$ for Daugman's algorithm and to $0.07 \%$ for the proposed fusion algorithm.

We extended the validation of proposed quality enhancement algorithm using the Miles, UBIRIS and the UPOL iris databases. In this validation we used three iris images for training. Results of the two iris recognition algorithms, Daugman's implementation and SVM based match score fusion, are shown in Table 5.3. The results show that the FRR of 


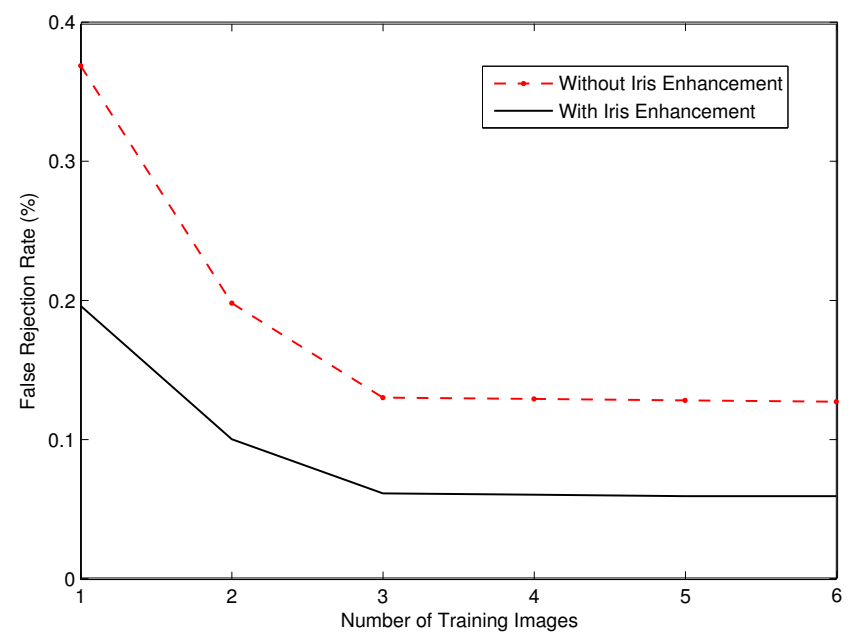

Figure 5.10: FRR vs Number of training samples for Daugman's implementation (on CASIA database)

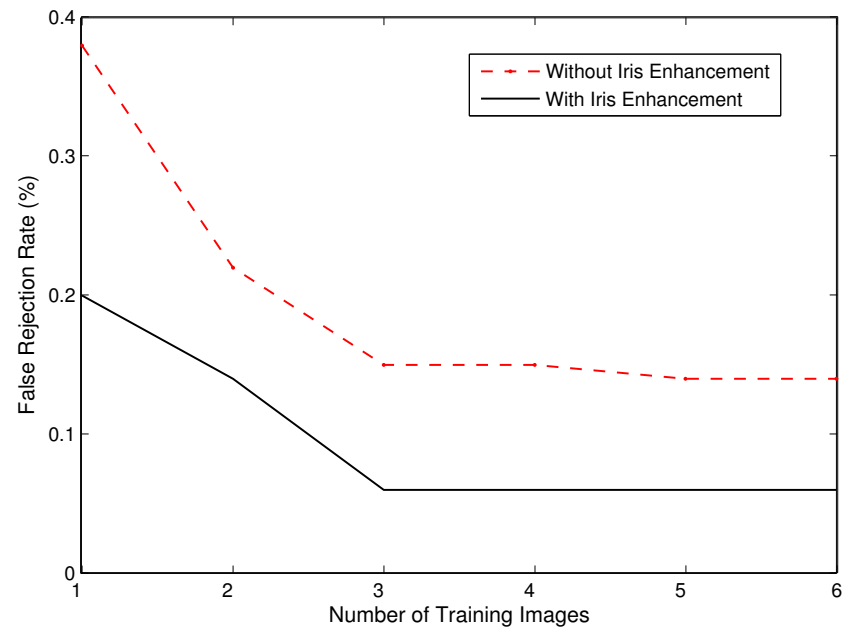

Figure 5.11: FRR vs Number of training samples for SVM based match score fusion (on CASIA database) 
Table 5.3: Performance validation of proposed iris enhancement algorithm on different databases (Number of training image $=3$ )

\begin{tabular}{|c|c|c|c|c|}
\hline \multirow[b]{2}{*}{ Database } & \multicolumn{2}{|c|}{ Daugman's Implementation } & \multicolumn{2}{|c|}{ SVM Based Match Score Fusion } \\
\hline & $\begin{array}{c}\text { FRR Without } \\
\text { Iris Enhancement }\end{array}$ & $\begin{array}{c}\text { FRR With } \\
\text { Iris Enhancement }\end{array}$ & $\begin{array}{c}\text { FRR Without } \\
\text { Iris Enhancement } \\
\end{array}$ & $\begin{array}{c}\text { FRR With } \\
\text { Iris Enhancement }\end{array}$ \\
\hline CASIA & $0.13 \%$ & $0.06 \%$ & $0.13 \%$ & $0.06 \%$ \\
\hline Miles & $0.10 \%$ & $0.05 \%$ & $0.10 \%$ & $0.05 \%$ \\
\hline UBIRIS & $6.32 \%$ & $3.75 \%$ & $6.41 \%$ & $3.76 \%$ \\
\hline UPOL & $0.08 \%$ & $0.04 \%$ & $0.08 \%$ & $0.04 \%$ \\
\hline
\end{tabular}

iris recognition algorithms is reduced by approximately $50 \%$ when used with the proposed quality enhancement algorithm and three images for training.

\subsection{Comparison with Existing Algorithms}

The proposed SVM based match score fusion algorithm (which fuses the textural and the topological features) was compared with [17], [46], and [64]. We implemented the three algorithms based on our interpretation of the algorithms presented in the research papers. Table 5.4 presents the comparison on the UBIRIS database using three images for training. Table shows that the algorithm proposed in this research performs better than the algorithms in [46] and [64] and performs comparable with Daugman's algorithm [19]. The high values of FRR are due to the poor quality of iris images as shown in Figure 5.8. However, experimental results show that the proposed algorithms are able to significantly reduce the false rejection rate. 
Table 5.4: Comparison of four iris recognition algorithms on UBIRIS database [56] using three images for training

\begin{tabular}{|c|c|c|c|c|}
\hline \hline & Localization & $\begin{array}{c}\text { Feature } \\
\text { Extractor }\end{array}$ & Matching & $\begin{array}{c}\text { FRR } \\
\text { (at 0 \% FAR) }\end{array}$ \\
\hline Daugman [17] & $\begin{array}{c}\text { Integro Differential } \\
\text { Operator }\end{array}$ & $\begin{array}{c}\text { 2D Gabor } \\
\text { Wavelet }\end{array}$ & $\begin{array}{c}\text { Hamming } \\
\text { Distance }\end{array}$ & $3.75 \%$ \\
\hline Ma et al. [46] & Hough & $\begin{array}{c}\text { Dyadic Wavelet } \\
\text { Transform }\end{array}$ & Exclusive OR & $5.18 \%$ \\
\hline Tan et al. [64] & Hough & $\begin{array}{c}\text { Multichannel } \\
\text { Transform }\end{array}$ & $\begin{array}{c}\text { FLD and } \\
\text { Sparial Filters }\end{array}$ & $6.32 \%$ \\
\hline Proposed & Elliptical & 1D log polar Gabor & SVM based \\
Boundary & and Euler Number & Match Score Fusion & $3.76 \%$ \\
\hline \hline
\end{tabular}




\section{Chapter 6}

\section{Conclusion}

The process of biometric identification/verification is currently a very active field of research. Many applications that require authentication are moving away from the use of passwords, PINs and cards, and are now adopting different biometric features. This is because of the higher degree of confidence achieved by using unique physical and/or behavioral biometric characteristics to identify a person. In literature, iris recognition is reported as one of the most reliable biometric authentication method. Despite these claims, high false rejection cases due to user inconvenience hinders the world wide use of iris recognition systems. One way to improve the performance of iris recognition systems is to reduce the false rejection rates without compromising the false acceptance rate.

In this thesis, our goal is to reduce the false rejection rate in iris biometrics. We proposed a novel iris segmentation algorithm for non-ideal iris images which is based on the concept that non-ideal iris images have elliptical shape. Using this segmentation algorithm, iris is segmented from the eye image and converted into polar coordinates. Further, we proposed an image quality enhancement algorithm using intelligent $2 \nu$-SVM based learning technique. In this process, we concurrently apply a set of selected enhancement algorithms to the whole image. Thus each image contains the enhanced local regions. These enhanced local regions are identified from each of the transformed images using the learning algorithm. Fusion of these high quality pixels is synergistically combined to form a single high quality and 
feature-rich iris image.

Next, we proposed the feature extraction algorithm which extracts the textural features from iris image using 1D log polar Gabor and the topological features using Euler numbers. 1D log polar Gabor wavelet is applied on a transformed polar iris image to extract the textural features and these features are encoded in the form of a binary iris template. Euler numbers are used to extract the topological features of an iris image and a 4-tuple Euler code is generated. Since Euler numbers are invariant to rotation, translation, scaling and rubber sheet transformation of the image, it gives a good way of representing the features of iris image. We also proposed three fusion schemes to fuse these two features at the match score level and the decision level. Learning based match score fusion algorithm uses SVM training to fuse the match scores of textural and topological features. First decision fusion algorithm is based on the empirical thresholds obtained after extensive experiments. Another fusion algorithm uses SVM based learning approach to obtain final decision using decisions obtained from textural and topological feature matching.

For validating the proposed algorithms, experiments are performed on four iris image databases, CASIA, Miles Research, UBIRIS, and UPOL iris databases. Experimental results show that the proposed algorithms reduce the false rejection rate of iris biometric significantly. We also showed that if a minimum of three iris images are used for training, then the false rejection rate is further reduced. A comparison of the proposed algorithm with some existing algorithms show that the performance of the proposed iris recognition algorithm is comparable with existing algorithms and has low failure to enroll rate. 


\section{References}

[1] J. F. Aguilar, J. O. Garcia, J. G. Rodriguez, and J. Bigun, "Kernel-based multimodal biometric verification using quality signals," Proceedings of SPIE Biometric Technology for Human Identification, Vol. 5404, pp. 544 - 554, 2004.

[2] J. M. H. Ali, and A. E. Hassanien, "An iris recognition system to enhance e-security environment based on wavelet theory," AMO - Advanced Modeling and Optimization, Vol. 5, No. 2, pp. 93 - 104, 2003.

[3] C. Sanchez-Avila, R. Sanchez-Reillo, and D. de Martin-Roche, "Iris recognition for biometric identification using dyadic wavelet transform zero-crossing," Proceedings of the 35th IEEE International Carnahan Conference on Security Technology, pp. 272 $277,2001$.

[4] C. Sanchez-Avila, and R. Snchez-Reillo, "Two different approaches for iris recognition using Gabor filters and multiscale zero-crossing representation," Pattern Recognition, Vol. 38, No. 2, pp. 231 - 240, 2005.

[5] E. Bigun, J. Bigun, B. Duc, and S. Fischer, "Expert conciliation for multimodal person authentication systems using bayesian statistics," Proceedings of the First International Conference on Audio and Video based Personal Authentication, 291 - 300, 1997.

[6] W. W. Boles, and B. Boashash, "A human identification technique using images of the iris and wavelet transform," IEEE Transactions on Signal Processing, Vol. 46, No. 4, pp. $1185-1188,1998$.

[7] P.-H. Chen, C.-J. Lin, and B. Schlkopf, "A tutorial on nu-support vector machines," Applied Stochastic Models in Business and Industry, Vol. 21, pp. 111 - 136, 2005.

[8] W.-S. Chen, and S.-Y. Yuan, "A novel personal biometric authentication technique using human iris based on fractal dimension features," Proceedings of the International Conference on Acoustics, Speech and Signal Processing, Vol. 3, pp. 201 - 204, 2003.

[9] H. G. Chew, C. C. Lim, and R. E. Bogner, "An implementation of training dual-nu support vector machines," In Qi, Teo, and Yang, editors, Optimization and Control with Applications, Kluwer, 2004. 
[10] C. - N. Chun, and R. Chung, "Iris recognition for iris tilted in depth," Proceedings of the International Conference on Computer Analysis of Images and Patterns: Lecture Notes of Computer Science, Vol. 2756, pp. 530 - 539, 2003.

[11] J. Cui, Y. Wang, J. Z. Huang, T. Tan, and Z. Sun, "An iris image synthesis method based on PCA and super-resolution," Proceedings of the 17th International Conference on Pattern Recognition, Vol. 4, pp. 471 - 474, 2004.

[12] A. Czajka, and A. Pacut, "Zak's transform for automatic identity verification," Proceedings of the 4th International Conference on Recent Advances in Soft Computing, pp. $374-379,2002$.

[13] C. H. Daouk, L. A. El-Esber, F. D. Kammoun, and M. A. Al-Alaoui, "Iris recognition," Proceedings of the 2nd IEEE International Symposium on Signal Processing and Information Technology, pp. 558 - 562, 2002.

[14] J. A. Dargham, A. Chekima, C. F. Liau, and W. Lye, "Iris recognition using selforganizing neural network," Student Conference on Research and Development, pp. $169-172,2002$.

[15] I. Daubechies, "Ten lectures on wavelets," CBMS-NSF Regional Conference Series in Applied Mathematics, 1992.

[16] J. G. Daugman "Complete discrete 2-d Gabor transforms by neural networks for image analysis and compression" IEEE Transactions on Acoustics, Speech, and Signal Processing, Vol. 36, No. 7, pp. 1169 - 1179, 1988.

[17] J. G. Daugman, "High confidence visual recognition of persons by a test of statistical independence," IEEE Transactions on Pattern Analysis and Machine Intelligence, Vol. 15, No. 11, pp. 1148 - 1161, 1993.

[18] J. Daugman, "Statistical richness of visual phase information," International Journal of Computer Vision, Vol. 45, No. 1, pp 25 - 38, 2001.

[19] J. Daugman, "The importance of being random: Statistical principles of iris recognition," Pattern Recognition, Vol. 36, No. 2, pp 279 - 291, 2003.

[20] V. Della, A. Michael, T. Chmielewski, T. A. Camus, M. Salganicoff, and M. Negin, "Methodology and apparatus for using the human iris as a robust biometric," Proceedings of the SPIE, Vol. 3246, pp. 65 - 74, 1998.

[21] M. Dobes, L. Machala, P. Tichavsky, and J. Pospisil, "Human eye iris recognition using the mutual information," Optik: International Journal for Light and Electron Optics, Vol. 115, No. 9, pp. 399 - 404, 2004.

[22] M. Dobes, and L. Machala, "Iris database," http://www.inf.upol.cz/iris/ 
[23] V. Dorairaj, N. Schmid, and G. Fahmy, "Performance evaluation of iris based recognition system implementing PCA and ICA techniques," Proceedings of the SPIE 2005 Symposium on Defense and Security, Conf. 5779: Biometric Technology for Human Identification II, 2005.

[24] B. Duc, G. Maitre, S. Fischer, and J. Bigun, "Person authentication by fusing face and speech information," Proceedings of the First International Conference on Audio and Video based Biometric Person authentication: Lecture Notes in Computer Science, pp. 311 - 318, 1997.

[25] D. J. Field, "Relations between the statistics of natural images and the response properties of cortical cells," Journal of the Optical Society of America, Vol. 4, pp. 2379 2394, 1987.

[26] D. J. Field, "Scale-invariance and self-similar wavelet transforms: An analysis of natural scenes and mammalian visual systems," In M. Farge, J. Hunt, and T. Vassilicos, Editors, Wavelets, Fractals and Fourier Transforms: New Developments and new applications, Oxford University Press, pp. 151 - 193, 1993.

[27] D. J. Field, "What is the goal of sensory coding?," Neural Computation, Vol. 6, pp. $559-601,1994$.

[28] P. A. Flach, "The geometry of ROC space: understanding machine learning metrics through ROC isometrics," Proceedings of the 20th International Conference on Machine Learning, pp. 194 - 201, 2003.

[29] L. Flom, and A. Safir, "Iris recognition system," United States Patent 4,641,349, 1987.

[30] H. Freeman, "Computer processing of line drawing images," Computer Surveys, Vol. 6, No. 1, pp. 57 - 97, 1974.

[31] B. Ganeshan, D. Theckedath, R. Young, and C. Chatwin, "Biometric iris recognition system using a fast and robust iris localization and alignment procedure," Optics and Lasers in Engineering, Volume 44, Issue 1, pp. 1 - 24, 2006.

[32] R. C. Gonzalez, and R. E. Woods, "Digital image processing," 2nd Edition, Prentice Hall, 2002.

[33] E. V. Gurianov, D. A. Zimnyakov, and V. A. Galanzha, "Iris patterns characterization by use of wiener spectra analysis: potentialities and restrictions," Proceedings of the SPIE, Vol. 4242, pp. 286 - 290, 2001.

[34] J. Huang, Y. Wang, J. Cui, and T. Tan, "Noise removal and impainting model for iris image," Proceedings of the International Conference on Image Processing, pp. 869 $872,2004$. 
[35] N. Seung-In, P. Kwanghuk, L. Chulhan, and K. Jaihie, "Multiresolution independent component identification," Proceedings of the International Technical Conference on Circuits/Systems, Computers and Communications, 2002.

[36] J. Jang, K. R. Park, J. Son, and Y. Lee, "Multi-unit iris recognition system by image check algorithm," Proceedings of the International Conference on Biometric Authentication: Lecture Notes in Computer Science, Vol. 3072, pp. 450 - 457, 2004.

[37] S. K. Kang, J. H. Min, and J. K. Paik, "Segmentation-based spatially adaptive motion blur removal and its application to surveillance systems," Proceedings of the International Conference on Image Processing, Vol. 1, pp. 245 - 248, 2001.

[38] P. J. N. Kapur, and A. K. C. Wong, "A new method for gray-level picture thresholding using the entropy of the histogram," Computer Vision Graphics and Image Processing, Vol. 29, pp. 273 - 285, 1985.

[39] J. Kittler, M. Hatef, R. P. Duin, and J. G. Matas, "On combining classifiers," IEEE Transactions on Pattern Analysis and Machine Intelligence, Vol. 20, No. 3, pp. 226 239, 1998.

[40] W. K. Kong, and D. Zhang, "Accurate iris segmentation based on novel reflection and eyelash detection model," Proceedings of the International Symposium on Intelligent Multimedia, Video and Speech Processing, pp. 263 - 266, 2001.

[41] A. Lefohn, B. Budge, P. Shirley, R. Caruso, and Erik Reinhard, "An ocularist's approach to human iris synthesis," IEEE Computer Graphics and Applications, Vol. 23, No. 6, pp. 70 - 75, 2003.

[42] S. Lim, K. Lee, O. Byeon, and T. Kim, "Efficient iris recognition through improvement of feature vector and classifier," Journal of Electronics and Telecommunication Research Institute, Vol. 23, No. 2, pp. 61 - 70, 2001.

[43] L. Ma, T. Tan, and Y. Wang., "Iris recognition based on multichannel Gabor filtering," Proceedings of the International Conference on Asian Conference on Computer Vision, pp. 1 - 5, 2002.

[44] L. Ma, T. Tan, and Y. Wang, "Iris recognition using circular symmetric filters," International Conference on Pattern Recognition, Vol. 2, pp. 414 - 417, 2002.

[45] L. Ma, T. Tan, D. Zhang, and Y. Wang, "Local intensity variation analysis for iris recognition," Pattern Recognition, Vol. 37, No. 6, pp. 1287 - 1298, 2004.

[46] L. Ma, T. Tan, Y. Wang, and D. Zhang, "Efficient iris recognition by characterizing key local variations," IEEE Transactions on Image Processing, Vol. 13, No. 6, pp. 739 - 750, 2004. 
[47] L. Machala, and J. Pospisil, "Alternatives of the statistical evaluation of the human iris structure," Proceedings of the SPIE, Vol. 4356, pp. 385 - 393, 2001.

[48] S. Makthal, and A. Ross, "Synthesis of iris images using markov random fields," Proceedings of the 13th European Signal Processing Conference, 2005.

[49] R. Malladi, and J. A. Sethian, "Image processing via level set curvature flow," Proceedings of the National Academy of Sciences, Vol. 92, No. 15, pp. 7046 - 7050, 1995.

[50] S. Mallat, "A wavelet tour of signal processing," 2nd Edition, Academic Press, 1999.

[51] B. R. Meena, M. Vatsa, R. Singh, and P. Gupta, "Iris based human verification algorithms," In Proceedings of the International Conference on Biometric Authentication, pp. 458 - 466, 2004.

[52] X. Ming, T. Xu, and Z. Wang, "Using multi-matching system based on a simplified deformable model of the human iris for iris recognition," Proceedings of the International Conference on Biometric Authentication: Lecture Notes in Computer Science, Vol. 3072, pp. 434 - 411, 2004.

[53] A. Muron, K. Petr, and P. Jaroslav, "Identification of persons by means of the fourier spectra of the optical transmission binary models of the human irises," Optics Communications, Vol. 192, pp. 161 - 167, 2001.

[54] C. Palm, and T. M. Lehmann, "Classification of color textures by Gabor filtering," Machine Graphics and Vision, Vol. 11, No. 2/3, pp. 195 - 219, 2002.

[55] K. Petr, A. Muron, and P. Jaroslav, "Human iris structure by the method of coherent optical fourier transform," Proceedings of the SPIE, Vol. 4356, pp. 394 - 400, 2001.

[56] H. Proena, and L. A. Alexandre, "UBIRIS: a noisy iris image database," Lecture Notes in Computer Science: 13th International Conference on Image Analysis and Processing, Vol. 1, pp. 970 - 977, 2005.

[57] R. M. Rao, and A. S. Bopardikar, "Wavelet transforms: introduction to theory \& applications," 1st Edition, Prentice Hall PTR, 1998.

[58] G. Ravichandran, and M. M. Trevedi, "Circular mellin filters for texture segmentation," IEEE Transactions on Image Processing, Vol. 4, No. 12, pp. 1629 - 1640, 1995.

[59] R. Roizenblatt, P. Schor, F. Dante, J. Roizenblatt, and R. Belfort, "Iris recognition as a biometric method after cataract surgery," Biomedical Engineering Online, Vol. 3, No. 2, 2004.

[60] E. Rydgren, E. A. Thomas, F. Amiel, F. Rossant, and A. Amara, "Iris features extraction using wavelet packets," Proceedings of the International Conference on Image Processing, Vol. 2, pp. 861 - 864, 2004. 
[61] D. G. Sheppard, K. Panchapakesan, A. Bilgin, B. R. Hunt, and M. W. Marcellin, "Removal of image defocus and motion blur effects with a nonlinear interpolative vector quantizer," Proceedings of the IEEE Southwest Symposium on Image Analysis and Interpretation, pp. 1 - 5, 1998.

[62] Z. Sun, Y. Wang, T. Tan, and J. Cui, "Improving iris recognition accuracy via cascaded classifiers," Proceedings of the International Conference on Biometric Authentication, Lecture Notes in Computer Science Vol. 3072, pp. 418 - 425, 2004.

[63] H. Sung, J. Lim, J. H. Park, and Y. Lee, "Iris recognition using collarette boundary localization," Proceedings of the International Conference on Pattern Recognition, Vol. 4, pp. 857 - 860, 2004.

[64] T. Tan, L. Ma, Y. Wang, and D. Zhang, "Personal identification based on iris texture analysis," IEEE Transactions on Pattern Analysis and Machine Intelligence, Vol. 25, No. 12, pp. 1519 - 1533, 2003.

[65] C. L. Tisse, L. Martin, L. Torres, and M. Robert: "Iris recognition system for person identification," Pattern Recognition in Information Systems, pp. 186 - 199, 2000.

[66] C. L. Tisse, L. Torres, and R. Michel, "Person identification technique using human iris recognition," Proceedings of the 15th International Conference on Vision Interface, pp. $294-299,2002$.

[67] V. N. Vapnik, "Statistical learning theory," Wiley-Interscience, 1998.

[68] V. N. Vapnik, "The nature of statistical learning theory," 2nd Edition, Springer, 1999.

[69] M. Vatsa, R. Singh, and P. Gupta, "Comparison of iris recognition algorithms," Proceedings of the International Conference on Intelligent Sensing and Information Processing, pp. $354-358,2004$.

[70] M. Vatsa, R. Singh, P. Mitra, and A. Noore, "Comparing robustness of watermarking algorithms on biometrics data," Proceedings of the Workshop on Biometric Challenges from Theory to Practice (ICPR Workshop), 2004.

[71] M. Vatsa, R. Singh, P. Mitra, and A. Noore, "Digital watermarking based secure multimodal biometric system," Proceedings of the IEEE International Conference on Systems, Man and Cybernetics, Vol. 3, pp. 2983 - 2987, 2004.

[72] P. Verlinde, G. Chollet, and M. Acheroy, "Multi-modal identity verification using expert fusion," Information Fusion Elsevier, Vol. 1, No. 1, pp. 17 - 33, 2000.

[73] B. V. K. Vijaya Kumar, C. Xie, and J. Thornton, "Iris verification using correlation filters," Proceedings of the International Conference on Audio and Video based Personal Authentication, pp. 697 - 705, 2003. 
[74] Y. Wang, T. Tan, and A. K. Jain, "Combining face and iris biometrics for identity verification," Proceedings of 4th International Conference on Audio and Video Based Biometric Person Authentication, pp. 805 - 813, 2003.

[75] R. P. Wildes, "Iris recognition: an emerging biometric technology," Proceedings of the IEEE, Vol. 85, No. 9, pp. 1348 - 1363, 1999.

[76] Y. Zhu, T. Tan, and Y. Wang, "Biometric personal identification based on iris patterns," Proceedings of the IEEE International Conference on Pattern Recognition, pp. $2801-2804,2000$.

[77] J. Zuo, and N. A. Schmid, "A model based, anatomy based method for synthesizing iris images," Proceedings of the International Conference on Biometric Authentication, 2006.

[78] Proceedings of International Conference on Audio and Video Based Personal Authentication.

[79] Proceedings of International Conference on Biometric Authentication, 2004.

[80] http://nlpr-web.ia.ac.cn/english/irds/irisdatabase.htm

[81] http://www.iridiantech.com/

[82] http://www.milesresearch.com/ 